\title{
Activités
}

10-2 | Octobre 2013

Intervenir sur le travail

\section{Ergonomie et domaine muséal}

Ergonomics and museums

Anne Bationo-Tillon

\section{OpenEdition}

Journals

Édition électronique

URL : http://journals.openedition.org/activites/752

DOI : 10.4000/activites.752

ISSN : 1765-2723

\section{Éditeur}

ARPACT - Association Recherches et Pratiques sur les ACTivités

\section{Référence électronique}

Anne Bationo-Tillon, «Ergonomie et domaine muséal », Activités [En ligne], 10-2 | Octobre 2013, mis en ligne le 15 octobre 2013, consulté le 01 mai 2019. URL : http://journals.openedition.org/activites/752 DOI : 10.4000/activites.752

\section{cc) () (을}

Activités est mis à disposition selon les termes de la licence Creative Commons Attribution - Pas d'Utilisation Commerciale - Pas de Modification 4.0 International 


\title{
Ergonomie et domaine muséal
}

\author{
Anne Bationo-Tillon \\ Université Paris 8, Laboratoire Paragraphe/équipe C3U, 2 rue de la liberté, 93526 Saint-Denis cedex \\ anne.bationo-tillon@univ-paris8.fr
}

\begin{abstract}
Ergonomics and museums. This paper suggests looking at the museum sector through concepts and methods which stem from ergonomics. We first set out the questions that the sector raises. We then propose a model for the activity of an encounter between a work of art and a capable subject visiting a museum (MARO). More precisely, we examine the various aspects of the different categories of situation which may occur when encountering a work of art. The paper presents the empirical results of two studies: a group museum tour and an individual museum visit with a device. We describe and compare the invariants of the visitors' activity with the MARO model. Finally, we discuss how the model helps us to better study and improve the design of museum tools for both visitors and museum professionals.
\end{abstract}

\section{KEYWORDS}

capable subjects, mediation, work of art in museum

\section{Introduction}

L'ergonomie a développé ses méthodologies et ses concepts théoriques depuis une cinquantaine d'années en se focalisant sur l'activité réelle de travail (Ombredane, \& Faverge, 1955). La recherche en ergonomie poursuit un double objectif contribuer au champ de connaissances des activités humaines situées tout en participant à la transformation du monde réel (Daniellou, 1996). Par ailleurs, le chercheur en ergonomie a un devoir de prospective qui doit se traduire par le choix d'études de situations encore rares ou d'aspects très particuliers de certaines activités (Falzon, 1998). L'objet de ce papier est donc d'éclairer des activités encore peu explorées dans le champ de l'ergonomie à savoir l'activité de découverte, de rencontre d'une œuvre d'art dans un contexte muséal. À cette fin, nous mobilisons les méthodes et concepts classiques issus du travail. Plus précisément, nous proposons un Modèle de l'Activité de Rencontre avec l'EEuvre en contexte muséal. Ce modèle que nous nommons (MARO) a émergé de nos analyses de situations réelles au Musée des Beaux-Arts de Rennes ainsi que de la confrontation au corpus de connaissances issues d'autres disciplines à ce sujet. Ainsi, nous considérons la cognition comme incarnée et située, et nous tenons à intégrer la singularité des sujets, la variabilité des situations afin d'éclairer les caractéristiques de cette activité muséale.

Nous effectuerons dans un premier temps un état de l'art du domaine muséal afin d'identifier les interrogations qui traversent ce champ et de positionner nos propres questionnements. Ceci nous amènera à préciser le cadre théorique de cette recherche ainsi que le modèle MARO qui a émergé au sein de nos travaux. Nous présenterons ensuite la manière dont ce modèle peut être mobilisé pour analyser des dispositifs de médiation culturelle à travers deux études empiriques. Pour finir, nous discuterons du statut de ce modèle dans le cadre d'une démarche ergonomique ainsi que pour les professionnels du musée. 


\section{1.- Le domaine muséal : un nouveau territoire pour l'ergonomie?}

Il existe une multitude d'études relatives au domaine muséal, celles-ci proviennent de disciplines variées et cherchent à éclairer des questions très différentes : la fréquentation des musées, la médiation culturelle, la muséographie, les aides à la visite, le comportement des visiteurs, etc. Depuis l'ergonomie, nous nous intéressons pour notre part à l'activité réelle des visiteurs en situation muséale et nous allons montrer que la plupart de ces études documentent cette question de manière fragmentaire.

\section{1.- Qui fréquente les musées ?}

Bon nombre d'études visent à fournir une taxonomie des visiteurs (âge, sexe, catégorie socioprofessionnelle) et du contexte social de la visite (seul, en famille, dans le cadre scolaire...) (Donat, 1998). Dans ce cas, l'analyse octroie une large part à l'appartenance sociodémographique des potentiels visiteurs, car il est supposé que la réception de l'œuvre d'art est conditionnée par la situation sociale des acteurs. La finalité de ces études est de connaître la population des visiteurs et de comprendre leurs besoins ainsi que les raisons pour lesquelles certaines personnes ne vont pas dans les musées (Hooper-Greenhill, 1994). C'est dans ce but que Pedler (2005) cherche à comprendre la morphologie sociale des groupes qui font cercle autour des œuvres d'art, en identifiant notamment les normes qui sont des critères de choix des œuvres d'art par les visiteurs en fonction de leur statut social et de leur niveau d'éducation.

Ces études cartographient les spécificités de la population des visiteurs en fonction de leur appartenance socioculturelle. Elles éclairent peu l'activité réelle des visiteurs en situation muséale.

\section{2.- Comment privilégier la rencontre du visiteur avec l'œuvre d'art ?}

Les services culturels, pédagogiques, éducatifs, et d'accueil des publics se sont multipliés en Europe au cours des années 1990-2000 (Fauche, 2008). Ces divers services ont pour mission de faire le lien entre la sphère des experts (historiens de l'art, conservateurs) et la sphère des visiteurs. Ils se déclinent principalement sous trois formes complémentaires : la médiation humaine (présence physique du médiateur) ; la médiation indirecte (présence de fiche d'aide, de dossiers pédagogiques, de supports audio, vidéo pour les potentiels visiteurs); la conception de projet de médiation en lien avec une exposition cible lorsque l'institution culturelle est suffisamment importante.

Des études cherchant à définir les contours professionnels de la médiation culturelle (Fauche, 1998 ; Peyrin, 2010, 2012) ont établi que le médiateur doit reprendre le discours énoncé par des spécialistes scientifiques et l'adapter pour les visiteurs. Le médiateur exerce donc une fonction de traduction qui requiert une formation mixte issue d'une part de l'histoire de l'art et d'autre part des usagers, c'est-à-dire les publics des musées dans leur diversité. Il existe un nombre important de travaux à destination des jeunes publics (Cohen, 1996 ; Rebetez, 1970), en revanche l'éducation muséale en direction des adultes reste encore un domaine à explorer et à structurer (Dufresne-Tassé, 2006). Le mouvement de la médiation ne renvoie pas au discours unique de la vérité, mais au discours pluriel du partage, le médiateur agit en tension entre l'œuvre et les récepteurs (Caillet, 1994). La médiation culturelle se donne pour objectif de permettre aux visiteurs une rencontre authentique avec les œuvres du musée, et de les aider à se saisir de l'œuvre pour élaborer du sens et nourrir leur rapport au monde (Dufresne-Tassé, 2006).

Cette littérature éclaire les missions, contradictions et pratiques de la médiation culturelle, et permet de bien comprendre ses particularités. En revanche, elle n'entre pas dans le détail de l'activité du visiteur. Nous chercherons pour notre part, à comprendre comment ces 
ressources de médiation culturelle (médiation humaine, médiation technique) sont mobilisées et/ou s'intègrent dans l'activité du visiteur.

\section{3.- Comment optimiser la muséographie ?}

Diverses recherches visent à concevoir des univers muséaux en adéquation avec le fonctionnement humain (Hooper-Grenhill, 1999). Ainsi la psychologie expérimentale cherche à identifier, via des études en laboratoire, les critères optimums de lisibilité (longueur et complexité des phrases) et visibilité des cartels, panneaux, catalogues pour que les visiteurs se repèrent facilement et intègrent les contenus présentés (Ekary, 1986; Belcher, 1991). D'autres études cherchent à comprendre la manière dont les textes de l'exposition sont intégrés dans les conversations des visiteurs (McManus, 1989).

Afin d'optimiser la muséographie, des évaluations sont parfois menées en laboratoire, en amont de l'exposition, pour tester des titres ou thèmes d'exposition ou encore pour évaluer les meilleures combinaisons possibles de présentation de la collection comme par exemple choisir d'associer un seul ou plusieurs objets de la même famille à un texte donné (Lockett, 1991). On parle alors d'évaluation formative pour désigner les études qui accompagnent la réalisation de l'exposition et d'évaluation sommative pour nommer celles qui interviennent une fois l'exposition ouverte au public (Edeilman, \& Roustan, 2007). Les évaluations sommatives cherchent le plus souvent à caractériser les comportements des visiteurs au sein du musée en recueillant des observables uniquement objectivables tels que le temps passé sur chaque œuvre. Cependant, il est difficile pour ce type d'études d'identifier les obstacles que rencontrent les visiteurs, ainsi que les besoins des visiteurs dans leur diversité.

Ces approches évaluatives ont le mérite d'exister, cependant elles prennent peu en compte la situation singulière et se privent du point de vue du sujet afin de comprendre de quelle manière, celui-ci mobilise les ressources de la muséographie au cours de la visite.

\section{4.- Quelle est l'efficacité des dispositifs techniques d'aide à la visite individuelle?}

L'audioguide est actuellement le dispositif le plus répandu parmi les outils techniques de médiation culturelle. La fonction de traduction est celle qui est la plus utilisée, suivie de près par les fonctions d'aide à la discrimination perceptive et à l'interprétation de l'œuvre. En revanche, l'audioguide semble être une entrave à la liberté de penser et de ressentir (Deshayes, 2002). Des linguistes ethnométhodologues ont remarqué que les visiteurs organisent les tours de parole avec l'audioguide de la même manière qu'ils le font entre humains, c'est-à-dire qu'ils peuvent s'interrompre lorsque l'audioguide se met à parler ils soulignent, complètent le discours de l'audioguide (Woodruff, Szymanski, Grinter, \& Aoki, 2002). Par ailleurs, bien que les visiteurs désirent avoir leur propre guide, ils apprécient l'expérience sociale partagée, ainsi l'écoute synchronisée est un facteur clef de l'interaction sociale. Cependant Fockler, Zeidler, Brombach, Bruns, et Bimber (2005) pointent le besoin d'aller au-delà des classiques guides audio qui ne permettent pas de présenter des graphiques, images, informations textuelles et qui de surcroit obligent le visiteur à repérer le numéro de l'œuvre au sein de l'exposition.

Damala, Cuhaud, Bationo-Tillon, Houlier et Marchal (2008) décrivent le nombre croissant de musées et de galeries proposant des dispositifs multimédias mobiles et montrent que le visiteur se retrouve souvent avec des documents de nature hétérogène (prospectus papier, guide multimédia). Il existe de plus en plus d'études d'évaluation de ces nouveaux dispositifs, cependant les méthodes d'analyse sont hétérogènes. Ainsi, certains auteurs mettent en évidence les problèmes d'utilisabilité (Liestol, 2009 ; Miyashita, Meier, Tachikawa, Orlic, Elbe, Scholz, et al, 2008). Enfin, quelques rares études s'attaquent à la question de l'utilité et de manière parcellaire, à notre sens, puisque ce sont toujours les mêmes dimensions qui sont éclairées, le plus souvent les interactions sociales et familiales (Wakkary, Hatala, Muise, 
Tanenbaum, Corness, Mohabbati, \& Budd, 2009) et plus rarement l'exploration de l'environnement muséal (Hope, Nakamura, Takahashi, Nobayashi, Fukuoka, et al, 2009).

Ainsi, plusieurs auteurs s'attellent aux questions d'utilisabilité et d'utilité, que nous connaissons bien dans le champ de l'ergonomie. Pour notre part, nous pensons qu'il serait pertinent de prolonger ces approches en abordant plus spécifiquement la question de la transformation de l'activité de visite par ces dispositifs en essayant de comprendre ce qu'ils empêchent, ce qu'ils privilégient. Cependant pour avancer dans cette voie, une première phase semble indispensable : elle consiste à proposer un modèle de référence de l'activité de visite.

\section{5.- Que font réellement les visiteurs d'un musée ?}

Il existe une différence entre l'expérience du musée d'un visiteur théorique et l'expérience d'un visiteur réel.

Le visiteur va, au cours de sa déambulation s'approprier, composer et négocier le contenu muséal, c'est en effet ce que soulignent Véron et Levasseur (1991). Ils proposent également une taxonomie révélant la diversité des stratégies (visiteurs fourmis, poissons, papillons et sauterelles). Chaque visiteur étant porteur d'intérêts, de motivations et d'attentes qui lui sont propres (Falk \& Dierking, 1992), les visiteurs s'autorisent dans les faits des écarts plus ou moins grands vis-à-vis de la visite telle qu'elle est prescrite par les concepteurs.

Les musées sont des environnements sociaux, les visiteurs se désengagent et se réengagent de manière alternative dans des activités individuelles et des activités partagées (Woodruff, et al., 2002). Ils observent les autres visiteurs pour les imiter, pour obtenir plus d'information et pour découvrir le sens et la signification des œuvres (Hindmarsch, Heath, \& Vom Lehn, 2005). La fatigue influe également sur le rythme de la visite, ainsi la première partie de la visite (environ 45 minutes) est intense, puis l'attention diminue, ils changent d'attitude, sont moins focalisés sur les œuvres et commencent à anticiper la visite de la boutique ou du lieu de restauration du musée.

Ces études qualitatives décrivent la visite muséale en situation en intégrant la diversité et la variabilité des visiteurs sans omettre la dimension sociale. Elles documentent donc des dimensions de l'activité réelle des visiteurs, mais ne proposent pas de modèle de l'activité globale. Précisons à ce stade que la diversité des finalités des visiteurs constitue un obstacle méthodologique et théorique que nous essayerons de contourner en nous focalisant sur les invariants de l'activité et en proposant un modèle de l'activité.

\section{6.- Comment se déroule la rencontre du visiteur avec une ouvre ?}

Hooper-Greenhill, Moussouri, Hawtorne et Riley (2001) montrent comment, face à une œuvre, le sens se développe à travers un processus dialogique et interactif entre les intentions des conservateurs et l'expérience interprétative des visiteurs. La construction de sens pour le visiteur passe par l'intégration des évènements immédiats et de l'expérience passée. Les visiteurs portent en outre une attention particulière à trois facettes de l'œuvre : la qualité visuelle (couleur, lumière, composition, forme, espace); le contexte socio-culturel (la scène représentée sur l'œuvre, les messages didactiques, politiques, la trajectoire de l'artiste, les autres œuvres de l'artiste ou de l'époque) et le processus de création de l'œuvre (les matériaux utilisés, la technique et le style de l'artiste).

Quant à Dewey (2010), il éclaire la dimension sensible de la rencontre avec une œuvre d'art. La rencontre avec l'œuvre lorsqu'elle advient, émeut, subjugue, calme et tranquillise le visiteur. Le visiteur ressent immédiatement un sentiment d'harmonie des éléments entre eux. En effet l'artiste est l'auteur de l'action qui engendre l'émotion et le visiteur se saisit ou non de cette émotion en s'immergeant plus ou moins dans cette œuvre.

Ces auteurs se rapprochent du cœur de nos préoccupations puisqu'ils abordent la dimension 
analytique ou sensitive de la rencontre avec l'œuvre d'art, mais ne décrivent pas en situation, la manière dont ces deux tendances coexistent. Il s'agira donc d'intégrer ces deux orientations au sein du modèle de l'activité de rencontre avec une œuvre d'art.

\section{7.- Quand est-ce que l'art fonctionne ?}

Comme le rappelle Goodman (1996) une œuvre n'est jamais assurée de fonctionner: cela dépendra des capacités et de l'attention du spectateur, de l'environnement des circonstances de la contemplation. En effet, Goodman rappelle qu'une œuvre d'art remplit sa fonction lorsqu'elle est implémentée, autrement dit exposée devant un public. Il propose de déplacer la question de «qu'est-ce que l'art? » en «quand y a-t-il art ? ». À son sens, l'œuvre d'art fonctionne dans la mesure où elle est comprise, où ce qu'elle symbolise est discerné et affecte la façon dont le visiteur organise et aperçoit un monde. Il souligne que ce que le visiteur voit d'une œuvre d'art dépend de ce qu'il y apporte. Cependant, il constate que la plupart des visiteurs ne savent pas voir. Il pense que le musée doit assister et développer la capacité de voir des visiteurs, encourager la perception affinée, accroitre la sensibilité et les capacités discriminatives des visiteurs, encourager le regard et l'écoute critique. Il insiste sur le fait que le développement d'une compétence donnée peut en accroitre ou en inhiber une autre. Autrement dit, le développement d'une capacité peut se faire au détriment d'une autre, en atrophier une autre. Pour résumer, selon Goodman la compréhension d'une œuvre implique des processus actifs et constructifs de discrimination, d'interrelation et d'organisation.

Ainsi pour Goodman, l'art existe que s'il y a rencontre avec un visiteur acteur en mesure de comprendre et de voir. Il identifie un certain nombre de capacités indispensables pour qu'une rencontre advienne sans les relier directement aux situations singulières. Nous chercherons donc à intégrer la dimension située de l'activité ainsi qu'à modéliser l'activité de rencontre avec une œuvre d'art lorsque l'art fonctionne.

\section{2.- Ancrages théoriques du visiteur capable}

Afin d'éclairer la manière dont les visiteurs mobilisent les ressources de la médiation culturelle dans leur activité de rencontre avec une œuvre d'art, nous utiliserons l'approche instrumentale (Rabardel, 1995). Le cadre théorique de Rabardel (1995, 2001, 2005) nous permettra également de décrire l'activité du visiteur capable afin de prolonger les propositions de Goodman (1996) provenant de la philosophie esthétique analytique.

\section{1.- L'activité instrumentée des visiteurs}

L'approche instrumentale propose des concepts qui permettent d'appréhender le contexte muséal en tant que situations d'activité instrumentée. L'instrument se différencie de l'artefact et constitue un mixte d'intériorité et d'extériorité puisqu'il tient à la fois du sujet et de l'artefact: un artefact matériel ou symbolique produit par le sujet ou par d'autres et un schème d'utilisation associé provenant de la construction propre du sujet ou de l'appropriation des schèmes sociaux déjà formés extérieurement à lui. Le visiteur est donc une personne qui dispose d'un ensemble de ressources internes et externes (ressources de la médiation culturelle) qu'il mobilise au sein de ses activités et qui donnent forme à ses différents rapports au monde: aux objets de l'activité, aux autres sujets et à lui-même (Rabardel, 2005). Rappelons, que pour Rabardel, l'activité instrumentée est toujours située. Les situations singulières ont une influence déterminante sur l'activité et sont organisées en classe de situation selon les critères du sujet (en fonction de l'objet de l'activité). Quant aux familles d'activité, elles regroupent des classes de situation ayant un même type de finalité générale (Rabardel \& Bourmaud, 2003). 


\section{2.- Le sujet capable}

Pour Rabardel, le pouvoir d'agir est toujours situé dans un rapport singulier au monde réel et dépend des conditions internes et externes au sujet qui sont réunies à un moment particulier (ressources disponibles, obstacles, etc.). Rabardel (2005) distingue pouvoir d'agir et capacité d'agir. Le pouvoir d'agir concerne la possibilité pour un visiteur dans une situation donnée de comprendre, voir, saisir une œuvre d'art, tandis que les capacités d'agir sont relatives à l'ensemble des ressources que le visiteur a élaborées au cours du temps grâce à l'activité constructive. Comme Goodman le rappelle, la rencontre avec une œuvre d'art ne se déploie pas toujours, notre parti pris est de décrire dans ce papier un modèle de l'activité de rencontre avec une œuvre d'art d'un visiteur capable, autrement dit un modèle de l'activité de rencontre avec une œuvre d'art lorsque cette activité se déploie.

Nous nous focalisons donc sur l'activité du visiteur et les ressources qu'il mobilise et qui donnent forme à sa rencontre avec l'œuvre de manière différenciée en fonction des classes de situation. Par conséquent, la rencontre avec l'œuvre d'art ainsi que les diverses ressources de médiation culturelle seront interrogées depuis la cohérence propre de l'activité de visite. Combiner les propositions de Goodman et les concepts de classes de situation et sujet capable de Rabardel nous amène à considérer que le visiteur est acteur de sa visite, et que son pouvoir d'agir est différent selon les situations. Pour éclairer le pouvoir d'agir du visiteur, il est nécessaire de le confronter à un modèle de l'activité du sujet capable.

Les questions qui en découlent sont les suivantes: quelles sont les différentes facettes potentielles de la rencontre d'un visiteur avec une œuvre d'art? Autrement dit quelles sont les différentes classes de situation de la rencontre d'un visiteur capable avec une œuvre d'art ? Pour répondre à cette question, nous allons identifier les classes de situation de la rencontre avec une œuvre d'art lorsqu'elle se déploie.

\section{3.- Émergence du modèle de l'activité}

Ce modèle de l'activité a émergé grâce à un enrichissement mutuel et progressif entre une revue de la littérature et de nombreuses observations [quatre séries d'expérimentations (Bationo-Tillon, Marchal \& Houlier, 2009, 2010, 2011 ; Folcher \& Bationo-Tillon, 2012)] et une soixantaine d'entretiens menés au sein du Musée des Beaux-Arts de Rennes pendant trois ans.

\section{1.- Contexte}

Nos questionnements proviennent de notre contribution en tant qu'ergonome au projet ANR GAMME $^{1}$ qui visait à développer un guide augmenté mobile pour les musées et expositions (Bationo Tillon, Marchal \& Houlier, 2009, 2010, 2011). La réalité augmentée est une technologie émergente qui permet de désigner des systèmes interactifs caractérisés par la présentation combinée en temps réel d'entités virtuelles et d'éléments physiques de l'environnement réel (Azuma, 1997). Les enjeux de ce projet étaient multiples. D'un point de vue technique, il s'agissait de développer de nouveaux algorithmes de calcul de pose. Pour le musée, c'était une opportunité intéressante pour élargir la palette des outils d'animation mis à disposition des visiteurs. Quant à notre objectif en tant qu'ergonome au sein de ce projet, il était double : d'une part, alimenter le corps de connaissance des activités humaines situées en l'occurrence les activités muséales et, d'autre part, contribuer à la conception de guide augmenté en injectant aux différentes étapes de la conception des connaissances relatives à l'activité. Nous avons donc déroulé les méthodologies classiques de l'ergonomie de langue française lorsqu'elle est confrontée au paradoxe de la conception (Theureau \& Pinsky, 1984).

1 Ce projet s'appuie sur un mixage équilibré d'entreprises (Le Musée des Beaux-Arts de Rennes, l'INRIA,

Movea, CEA, Orange Labs) et de domaines de compétences (informatique, graphisme, ergonomie...). 
Il s'agit d'identifier les situations de référence ou d'actions caractéristiques (Daniellou \& Beguin, 2004), et d'en faire émerger les invariants (Rabardel, 1995), pour alimenter le processus de conception dès les phases amont. Ensuite, nous nous sommes inspirée de la démarche ergonomique appliquée au champ muséal telle que décrite par Bach, Salembier et Dubois (2007). Tout d'abord, l'analyse initiale du contexte qui se concrétise par le biais de réunions avec les différents acteurs (curateurs du musée, spécialistes des systèmes mixtes, graphistes, etc.) du projet. Cette phase permet de préciser la thématique muséographique, les principes fonctionnels et de définir l'interaction. Ensuite, dès les premières phases de maquettage peuvent avoir lieu des expérimentations en labo ou in vivo de manière itérative.

Nous nous centrons au sein de ce papier sur la description des étapes qui nous ont permis de mettre en œuvre le modèle de l'activité muséale : à savoir les situations de référence et les expérimentations intégrant le prototype de guide augmenté.

\section{2.- Le modèle de la rencontre avec une œuvre d'art d'un visiteur capable}

Ce modèle se réfère à l'activité potentielle d'un visiteur capable. Il ne correspond pas à l'activité telle qu'elle se réalise dans chacune des situations singulières, mais plutôt à la potentialité de l'activité lorsque la rencontre avec une œuvre d'art se déploie sous toutes ses facettes. Ce modèle de l'activité a émergé à l'issue d'un aller-retour entre une revue de la littérature et des observations empiriques variées et répétées au cours de trois années de collaboration avec le Musée des Beaux Arts de Rennes. Ce modèle sédimente donc notre compréhension de l'activité de rencontre avec une oeuvre.

Le modèle de l'activité muséale de rencontre avec une œuvre d'art (MARO) est composé de deux familles d'activité : l'activité sensitive et l'activité analytique. Les deux attitudes d'être au monde que décrit Merleau-Ponty (1945) renvoient à la distinction entre activité sensitive et activité analytique. L'activité sensitive correspond à une manière d'être au monde où la perception de l'œuvre peut se faire sans être enfermée dans une perspective. Le visiteur s'ancre dans l'œuvre, fixe l'œuvre, mais cet arrêt du regard n'est qu'une modalité de son mouvement puisque, il continue à l'intérieur de l'œuvre. Le visiteur ouvre l'œuvre, il regarde l'œuvre en s'enfonçant en elle, l'œuvre est inachevée, ouverte, elle est toute entière étalée. Lors de l'activité analytique, au lieu d'être approchée, saisie et engloutie par le sujet, l'œuvre est maintenue à distance et fait tableau devant le sujet.

Chacune de ces deux familles d'activité contient trois classes de situation que nous détaillons ci-dessous, en présentant des extraits de verbalisations ou d'observations de visiteurs au contact d'une même œuvre, l'œuvre de Rubens : «La chasse au tigre » (voir Figure 1).

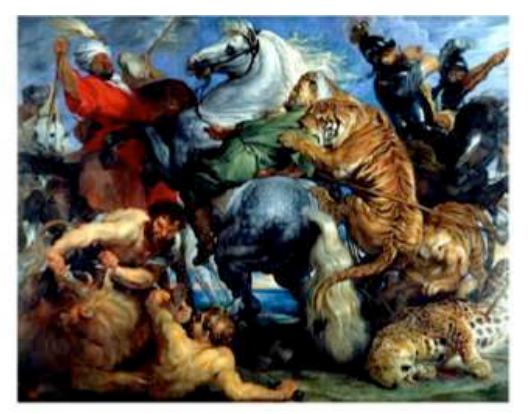

Figure $1:$ tableau de Rubens : La chasse au tigre (cette œuvre appartient au Musée des Beaux-Arts de Rennes)

Figure 1: Rubens painting: The Tiger hunt (this work of art belongs to Fine Art Museum of Rennes 
L'activité sensitive consiste pour le visiteur à faire émerger ses sensations, ses impressions au contact de l'œuvre. En effet, Dewey (2010) précise qu'un tableau est vivant, que l'art émeut, subjugue, calme, tranquillise. L'activité sensitive est composée de trois classes de situations :

— L'immersion qui correspond à l'émergence du ressenti du visiteur ici et maintenant. Il s'agit d'une situation englobante pour le visiteur qui plonge dans l'œuvre ici et maintenant. Il sollicite tous ses sens pour ressentir l'œuvre. Un des visiteurs nous a fait part de sa "sensation de courbe» face au Rubens, il s'agit donc de ses sensations lorsqu'il s'immerge dans l'œuvre. L'objet de l'activité du visiteur consiste à être à l'écoute de ses sensations au contact de l'œuvre.

- L'imprégnation qui rend compte des résonnances entre le ressenti éprouvé au contact de l'œuvre et ce même ressenti éprouvé dans d'autres situations de la vie quotidienne. Dewey (2010) évoque les échos de l'œuvre qui peuvent se répercuter en cycle en interagissant avec la totalité de notre expérience. À notre sens cette imprégnation peut avoir lieu sur place au sein du musée, mais également continuer après la visite. Il s'agit de ce que l'œuvre réveille en nous. C'est ce qui arrive à la jeune visiteuse qui évoque le divorce de ses parents face à la Chasse au tigre de Rubens. Dans cette optique, l'art participe à la domestication de notre espace intérieur (Tisseron, 2005). La peinture ouvre la possibilité pour un visiteur de dialoguer avec lui-même (Passeron, 1974). L'objet de l'activité du visiteur consiste à relier le fragment de vécu au contact de l'œuvre avec d'autres fragments de vécus.

— L'imagination qui correspond au prolongement possible par le visiteur. Goodman (1996) précise en effet, qu'un tableau est vivant et que ce que nous y voyons dépend de ce que nous y apportons. Dans cette perspective, l'activité sensitive ne peut se réduire à l'observation globale de ce qui est visible, c'est dans le même temps écouter son ressenti et observer au-delà du visible en imaginant, en déformant les images premières (Bachelard, 1943). C'est ce que fait la visiteuse qui imagine la scène au-delà du cadre spatio-temporel de l'œuvre figée qui lui fait face, lorsqu'elle imagine les cris des animaux, les mouvements, la scène vivante du tableau. L'objet de l'activité du visiteur est de prolonger l'œuvre, continuer l'œuvre en renouvelant son regard sur l'œuvre.

L'activité analytique consiste pour le visiteur à parcourir l'œuvre, la morceler, l'objectiver et la rendre catégorisable et reliable à divers domaines d'activité.

— La description fine de l'œuvre consiste à considérer la singularité et le détail de l'œuvre ici et maintenant, à identifier les divers éléments, à séparer le tout complexe de l'œuvre en parties, à distinguer les traits discriminants d'une œuvre (Goodman, 1996). Cette exploration active de l'œuvre permet de cerner les caractéristiques de l'œuvre (couleur, lumière, composition, formes). C'est ce que nous décrit cette visiteuse de 21 ans, «Là je vais voir les détails, je vais voir les animaux, les expressions » ou encore cette visiteuse de 16 ans "Là je m'avance pour regarder les bébés tigres, je vais voir ce qui m'intéresse puis je me recule». L'objet de l'activité du visiteur consiste à parcourir, découper et nommer les éléments du tableau.

— La distanciation de l'œuvre consiste à considérer l'œuvre de visu pour créer des liens entre les éléments de l'œuvre et les domaines précis de prédilection du visiteur. Le visiteur se déprend de l'œuvre singulière, il l'objective en la regardant de visu. Il s'agit d'une distance critique où l'œuvre est réifiée, stabilisée pour pouvoir être associée à d'autres champs de connaissances, d'autres domaines d'activités selon la préoccupation du visiteur (Tisseron, 2005). Ce domaine d'activité peut concerner l'histoire de l'art et ses concepts, mais également le contexte historico-culturel de la production de l'œuvre tout comme le processus de genèse de l'œuvre. Passeron (1974) rappelle que voir une œuvre, c'est voir les gestes que l'on ferait pour la réaliser. C'est d'ailleurs ce que fait un des visiteurs observés «Alors là le Rubens, je me rapproche, je m'avance pour regarder les 
brillants du cheval, et je balance un regard sur le tableau de Crayer (comme les deux peintres sont liés pour voir comment il avait fait, je compare les brillants sur le crin de cheval)». L'objet de l'activité du visiteur consiste à intégrer les éléments identifiés dans des domaines de connaissances stabilisés.

— L'émergence de questions : au contact de l'œuvre, des questions peuvent surgir chez le visiteur. Un des visiteurs nous a fait part des interrogations qui le traversent lorsqu'il regarde l'œuvre de Rubens "Je me demande si Rubens a été témoin de ce type de chasse ». Ces questions sans réponses immédiates ouvrent un espace à défricher dans le futur. Bachelard (1971) précise en effet que toute connaissance est une réponse à une question. Certains visiteurs projettent d'aller chercher des éléments de réponse sur Internet, d'autres seront moins proactifs, mais lorsqu'ils croiseront ce thème dans une autre situation, ils seront avides d'en savoir plus. L'objet de l'activité du visiteur consiste à formuler ses étonnements, interrogations au contact de l'œuvre.

Ces différentes classes de situation peuvent être concomitantes ou séquentielles, elles peuvent ne pas se déployer toutes et restent sous la dépendance d'un ensemble de déterminants relatifs aux œuvres, aux visiteurs, car comme le rappelle Goodman, une œuvre n'est jamais assurée de fonctionner, cela dépendra des capacités et de l'attention du spectateur, de l'environnement, des circonstances de la contemplation. Ce modèle constitue un outillage conceptuel qui rend compte de l'activité du visiteur capable. En le mobilisant pour décrire l'activité réelle d'un visiteur dans une situation singulière, nous pouvons rendre compte de l'enchainement des classes de situation au sein de séquences d'activité et révéler l'absence de classes de situation ou encore les enchainements répétés de certaines classes de situation.

\section{4.- Études empiriques ou la mise à l'épreuve du modèle}

Dans cet article, nous privilégions deux situations d'observation que le modèle de l'activité du visiteur capable (MARO) permet d'éclairer :

- une situation d'activité de visite muséale dans un contexte de médiation culturelle humaine et collective. Nous avons choisi des situations de références relativement structurées puisque nous nous sommes centrées sur des visites thématiques dispensées par les animatrices du musée. Notre hypothèse implicite étant que les animatrices sont au plus près des visiteurs et qu'elles les aident à déployer les facettes de la rencontre avec l'œuvre. Nous montrerons de quelle manière le modèle permet de comprendre l'articulation entre l'activité des animatrices et l'activité des visiteurs.

— une situation d'activité de visite muséale dans un contexte de médiation culturelle technique et individuelle, il s'agissait des expérimentations où des visiteurs utilisaient un prototype augmenté en situation réelle. Notre hypothèse implicite était que ce nouvel artefact en perturbant l'activité nous permettrait d'identifier des situations où il constitue une ressource pour augmenter le pouvoir d'agir du visiteur ainsi que des situations où il se révèle être un obstacle en limitant le pouvoir d'agir du visiteur. Nous montrerons de quelle manière le modèle de l'activité du visiteur capable nous permet d'étudier l'impact de ce dispositif sur l'activité de visite.

\section{1.- Activité de visite muséale et médiation culturelle humaine et collective}

\subsection{1.- Objectifs}

En choisissant des situations de référence déjà très organisées où l'activité des visiteurs est en grande partie déterminée par les interventions des animatrices, notre objectif est de confronter le modèle de l'activité de rencontre avec une œuvre (MARO) à la pratique des animatrices. 


\subsection{2.- Méthodologie}

Une dizaine de sessions d'observations de l'activité de visites thématiques ont été menées au sein du Musée des Beaux-Arts de Rennes afin d'identifier les caractéristiques de l'activité de visite ainsi que les caractéristiques de l'activité d'animation. Les visites thématiques consistent à accueillir des petits groupes d'une dizaine de personnes maximum et à les accompagner dans une découverte approfondie d'environ 6 œuvres de l'exposition sélectionnées en fonction de la thématique. Chaque session durait environ une heure. Il s'agissait d'une observation de type ethnographique dans la mesure où l'ergonome prenait des notes en accordant une attention particulière à la manière dont l'animatrice présentait les œuvres aux visiteurs, les ressources mobilisées, ainsi que la manière dont les visiteurs et les animatrices interagissaient. Ces sessions d'observation ont volontairement porté sur des populations de visiteurs variés (jeunes enfants, collégiens, jeunes adultes et adultes) ainsi que sur des groupes animés par trois animatrices différentes. Chaque session a donné lieu à un enregistrement audio puis a été entièrement transcrite. L'analyse de l'activité des visites thématiques nous a permis d'identifier un invariant de l'activité d'animation ainsi que des stratégies et des critères organisateurs de l'activité des animatrices transverses aux différentes visites et donc du même coup de l'activité de découverte des œuvres par les visiteurs. À l'issue de ces observations, nous avons confronté nos résultats (séquences d'activité et critères organisateurs de l'activité d'animation) aux animatrices au cours d'entretiens individuels afin de valider, nuancer ou enrichir nos analyses. Ces entretiens d'autoconfrontation ont été enregistrés puis retranscrits.

\subsection{3- L'activité des animatrices au regard de l'activité des visiteurs}

Nous évoquons d'abord les déterminants de l'activité des animatrices, puis nous présentons un invariant de l'activité d'animation qui se décompose en trois séquences d'activité. Enfin nous confrontons ces séquences d'activité au modèle MARO.

\section{Les déterminants de l'activité des animatrices}

Aurélie Peyrin (2010) s'est intéressée à la place du médiateur dans l'institution muséale. Elle rend compte de la diversité des conditions de travail (vacataires, contractuels ou fonctionnaires) et présente le métier de médiateur comme une vocation qui nécessite de s'approprier les connaissances des experts (commissaires d'expositions) et de s'adapter à des publics très diversifiés. Nous précisons donc les caractéristiques des médiatrices et de leur situation de travail au Musée des Beaux-Arts de Rennes avant de décrire plus précisément ce que nous avons appris de l'analyse de leur activité.

Les trois animatrices travaillent au Musée des Beaux-arts de Rennes depuis une vingtaine d'années et sont fonctionnaires. Elles ont été embauchées avec un an de décalage les unes des autres. Elles ont une formation en histoire de l'art pour deux d'entre elles et une formation en médiation pour la troisième. Le collectif de travail s'est élaboré au fil des années et fonctionne bien, elles ont co-construit un point de vue commun sur l'activité de médiation: "ce qui est intéressant, c'est que quand on a une difficulté dans un groupe de visite, on en parle toutes les trois pour savoir comment dépasser, on se dit que la difficulté vient toujours de nous et pas forcément des visiteurs et du groupe ». Elles redéfinissent leur mission de médiation en mettant l'accent sur l'importance de partir de ce que ressentent les personnes : «on leur demande qu'est-ce que vous éprouvez?... et ce qu'éprouvent les gens, ça ne se discute pas... par contre, on peut se questionner sur les raisons. Qu'est-ce qui fait qu'on peut éprouver cela dans cette image? » Elles revendiquent une démarche qui part toujours de l'expérience sensible: "Quand on reçoit des étudiants pour des vacations de médiation, la première chose qu'on leur dit c'est qu'il va falloir qu'ils soient dans une autre démarche que la démarche universitaire qui part plutôt du général pour aller vers le particulier et nous notre singularité, c'est qu'on a des æeuvres, et on est avant tout dans le sensible.»Elles s'appuient sur ce qui se passe dans les interactions avec les visiteurs (froncements de sourcils, sourires, questions) pour réajuster, modifier les ateliers au fil de 
l'eau «au fur et à mesure, on fait des expériences avec les visiteurs, ce sont eux qui nous incitent à trouver des solutions, ce sont eux qui nous guident ». Elles précisent enfin qu'elles ne sont pas là uniquement pour promouvoir le discours du commissaire d'exposition, mais qu'elles cherchent à se l'approprier suffisamment pour faire saisir aux visiteurs qu'il ne s'agit pas d'une juxtaposition d'œuvres.

\section{Un invariant de l'activité d'animation}

Tout d'abord, les animatrices introduisent chacune des œuvres de la visite thématique en suivant la plupart du temps la même trame qui se déroule en 3 séquences. Elles amènent les visiteurs à avoir une vision globale de l'œuvre dans un premier temps [séquence 1 qui dure environ 3 minutes] avant de leur faire détailler l'œuvre [séquence 2 qui dure environ 3 minutes] pour finir par repositionner l'œuvre dans un contexte plus large [séquence 3 qui dure environ 5 minutes]. Plus précisément, il s'agit d'un invariant de l'activité d'animation qui s'organise de la manière suivante :

— L'animatrice introduit d'abord l'œuvre de manière à ce que les visiteurs s'immergent dans l'œuvre, se posent des questions génériques (Quand? Qui ? Quoi ?, etc.). L'animatrice s'applique donc à faire émerger le ressenti et la perception globale des visiteurs face à l'œuvre. Lors des entretiens, une des animatrices nous a précisé "C'est d'abord important d'avoir une vision globale avant de s'arrêter sur un petit point. Nommer des choses dans leur ensemble, puis aller dans le plus profond.» Les animatrices nomment cette première étape l'observation globale.

- Dans un second temps, elle pousse le visiteur à décrire finement l'œuvre via divers dispositifs pour que le visiteur accède à la singularité de cette œuvre. Les animatrices appellent cette phase description fine.

- Puis dans un troisième temps, elle effectue avec les visiteurs, en partant de leur référentiel une analyse plus distancée avec une éventuelle introduction de concepts techniques, d'histoire de l'art, de courants artistiques, en les incitant à formuler leurs questions. Les animatrices identifient cette phase comme une phase d'analyse.

Le tableau 1 confronte l'organisation de l'activité des animatrices selon ces 3 étapes et les classes de situation de l'activité des visiteurs. 


\begin{tabular}{|c|c|c|}
\hline \multicolumn{2}{|c|}{ Séquences d'activité des animatrices } & Classes de situation de l'activité \\
\hline 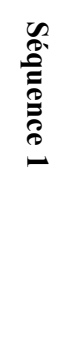 & $\begin{array}{l}\qquad \text { FAIRE OBSERVER GLOBALEMENT } \\
\text { QUESTIONNER : L'animatrice questionne le ressenti, le } \\
\text { référentiel des visiteurs sur l'œuvre, l'animatrice pose des } \\
\text { questions ouvertes sans réponse évidente, elle pose des questions } \\
\text { relatives à l'imagination du public autour de l'œuvre } \\
\text { IMPLIQUER : L'animatrice implique le visiteur en le faisant } \\
\text { mimer des sentiments } \\
\text { critères : ne pas assener une seule interprétation, placer le } \\
\text { ressenti du visiteur au centre }\end{array}$ & $\begin{array}{l}\text { ACTIVITÉ SENSITIVE } \\
\text { - immersion : plonger dans } \\
\text { l'œuvre, en écoutant son ressenti, } \\
\text { en s'exprimant, en mimant des } \\
\text { sentiments } \\
\text { - imprégnation : Observer et } \\
\text { exprimer les échos que l'œuvre } \\
\text { provoque en soi } \\
\text { - imagination : percevoir la scène } \\
\text { au-delà de l'image première }\end{array}$ \\
\hline 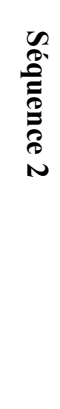 & $\begin{array}{l}\text { FAIRE DECRIRE FINEMENT } \\
\text { MONTRER : l'animatrice attire l'attention du public via des } \\
\text { déictiques corporels, langagiers, la prosodie, la théâtralité, le } \\
\text { silence, l'ordre de présentation. } \\
\text { IMPLIQUER : l'animatrice implique le visiteur en le faisant } \\
\text { mimer les positions des personnages, en le faisant décrire } \\
\text { finement. Elle peut inviter le visiteur à se déplacer vis-à-vis de } \\
\text { l'œuvre. } \\
\text { Critères : entrer dans la singularité de l'œuvre, ne pas } \\
\text { considérer l'œuvre comme une illustration d'un courant } \\
\text { artistique... }\end{array}$ & $\begin{array}{l}\text { ACTIVITÉ ANALYTIQUE } \\
\text { - description fine : Regarder de } \\
\text { manière prolongée en détaillant } \\
\text { l'œuvre, distinguant, s'avançant } \\
\text { puis se reculant, en mimant les } \\
\text { positions des personnages }\end{array}$ \\
\hline 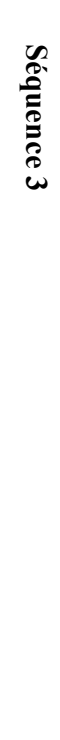 & $\begin{array}{l}\text { FAIRE ANALYSER } \\
\text { QUESTIONNER : l'animatrice questionne les visiteurs sur le } \\
\text { rapport de l'auteur et de l'œuvre (exemple : est-ce une allégorie, } \\
\text { une scène vue ? une scène réelle ?) } \\
\text { OUTILLER : introduire des concepts d'histoire de l'art, } \\
\text { désamorcer les idées reçues des visiteurs } \\
\text { IMPLIQUER : L'animatrice implique le visiteur en faisant faire } \\
\text { via les livrets pédagogiques, via des jeux de rôle } \\
\text { DÉCLOISONNER/RELIER : l'animatrice fait dialoguer une série } \\
\text { d'œuvres, une œuvre et d'autres ressources (Cartes } \\
\text { géographiques, lettres d'écrivains, ouvrages d'histoire de l'art,) } \\
\text { pour repositionner une œuvre dans l'histoire de l'art, dans un } \\
\text { contexte historique, sociologique, artistique (littérature, musique } \\
\text { époque)-relier l'œuvre avec les thèmes de la vie quotidienne avec } \\
\text { d'autres œuvres, d'autres styles, } \\
\text { Critères : relier l'œuvre à un contexte plus général, montrer } \\
\text { que l'œuvre est le fruit d'une série de choix artistiques } \\
\text { ÉCOUTER LES VISITEURS } \\
\text { Critères : Ne pas assener une interprétation définitive }\end{array}$ & $\begin{array}{l}\text { ACTIVITÉ ANALYTIQUE } \\
\text { - distanciation : deviner, } \\
\text { reconnaître, comparer, écouter, } \\
\text { déconstruire, créer des liens, } \\
\text { dessiner, sélectionner, choisir, } \\
\text { écouter, comparer, relier l'œu uvre } \\
\text { avec d'autres domaines, relier } \\
\text { avec d'autres connaissances, } \\
\text { situer l'œuvre dans un contexte, } \\
\text { reconnaître, }\end{array}$ \\
\hline
\end{tabular}

Tableau 1 : Les séquences d'activité des animatrices versus les classes de situation de l'activité des visiteurs muséaux

\section{Table 1: Curator activity versus categories of situation for museum visitor activity}

L'activité des animatrices consiste donc à aider le visiteur à occuper les différentes classes de situation du modèle de l'activité. Dans cette optique, elles utilisent différentes ressources que nous résumons dans le figure 2 ci-après. Ce dernier précise les deux familles d'activité (lecture verticale), ainsi que les trois classes de situation pour chaque famille d'activité qui consistent à se confronter à l'œuvre, ici et maintenant, puis à relier l'œuvre puis à prolonger l'œuvre (lecture horizontale). 


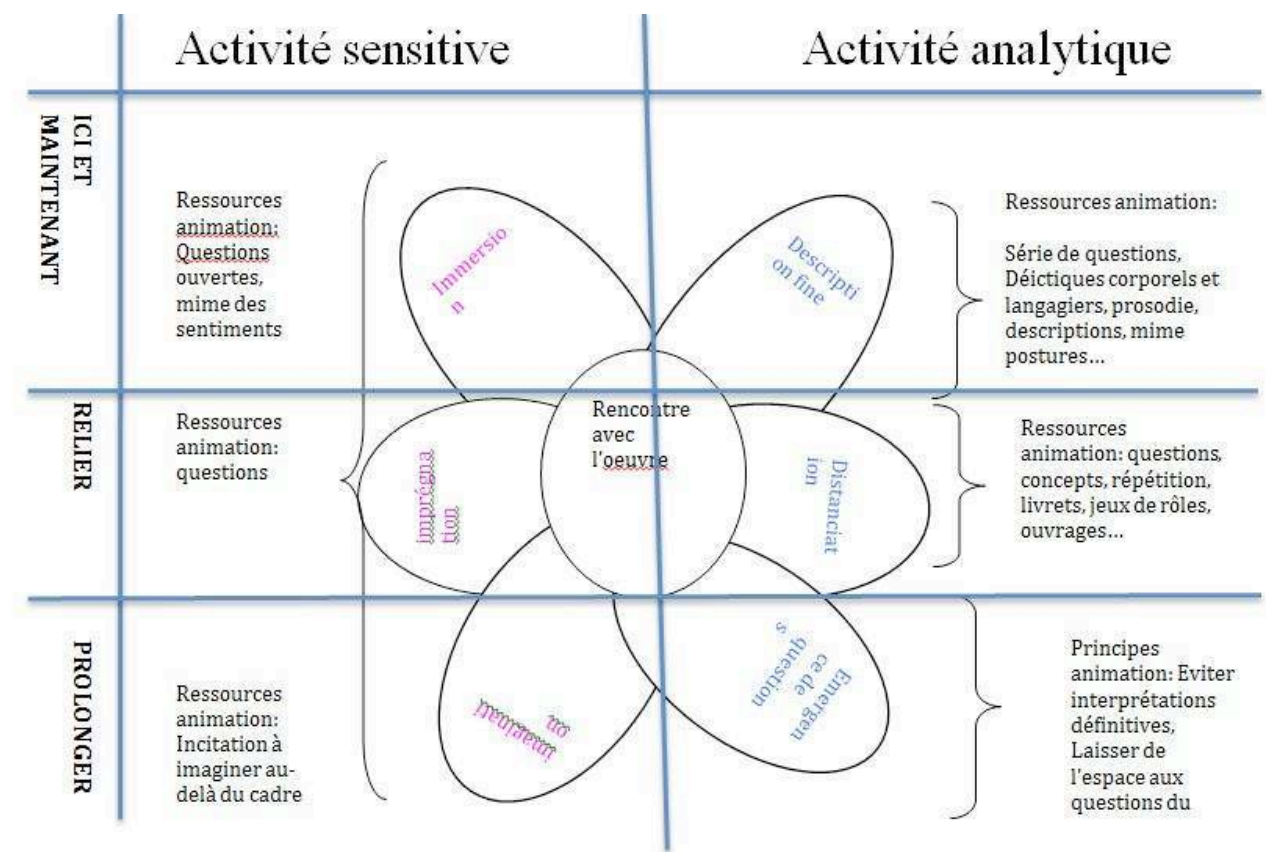

Figure 2 : Ressources des animatrices mobilisées pour aider le visiteur à occuper les différentes classes de situation

Figure 2: Resources mobilized by curators to help visitors occupy the different categories of situation

Nous allons au sein de la section suivante confronter chacune des séquences avec les classes de situation du modèle de l'activité de rencontre avec une œuvre (MARO). Nous illustrerons nos propos à l'aide des observations de visites thématiques et verbalisations des animatrices issues des entretiens d'auto-confrontation.

\section{Séquences d'activité des animatrices et classes de situation de l'activité des visiteurs}

\section{Séquence 1 versus ACTIVITÉ SENSITIVE :}

Afin de faire observer globalement l'œuvre durant la séquence 1, les animatrices mobilisent différents types de ressources : des questions ouvertes sans réponses évidentes qui peuvent être relatives au référentiel et ressenti (a), ou encore qui sollicitent l'imagination des visiteurs (b), des jeux de mimes de sentiments afin de faire entrer le visiteur dans le tableau comme nous l'ont précisé les animatrices.

« Nous on part de leur bagage, car ils savent beaucoup de choses et on veut adapter leur savoir à ce qu'on veut leur faire apprendre, on part toujours du public, il sait beaucoup de choses »

«Je les interroge ensuite: qu'est-ce que vous avez remarqué ? Qu'est-ce que vous avez ressenti ? Vos premières impressions ? Et à partir de là, ça peut se construire donc du coup »

(a) Extrait entretien auto-confrontation animatrice

L'animatrice est debout devant un tableau qui représente trois indiens avec de la fumée, elle demande aux enfants «Imaginez une scène en dehors du tableau qui serait un des tableaux en face ». Deux enfants répondent l'un après l'autre spontanément «Une scène de chasse d'animaux ! »- « Des cowboys arrivent »

(b) Extrait d'observation d'une animatrice avec des élèves de collège

Cependant, les animatrices pointent également des obstacles qu'elles doivent contourner lors de l'observation globale, elles sont attentives à ne pas catégoriser d'emblée l'œuvre présentée avant de faire émerger le ressenti des visiteurs, tout comme elles sont attentives à laisser certaines questions ouvertes sans réponses définitives. 
«Je ne vais pas dire le mot portrait au départ, ... c'est juste du ressenti et juste rentrer dans une image et une fois qu'on a bien décrypté tout ce qui se passe dans cette image, sculpture ou peinture et bien on va pouvoir leur dire ben voilà, ça c'est un portrait. »

(c) Extraits d'entretien d'auto-confrontation animatrice

Il ressort des entretiens d'auto-confrontation (c) que lors de cette séquence d'activité [Observation globale] les animatrices octroient une place importante à l'activité sensitive des visiteurs, sans distinguer plus finement les postures d'immersion, d'imprégnation et d'imagination. Autrement dit, ces classes de situation ne sont pas des catégories mobilisées explicitement et systématiquement dans leurs pratiques, cependant en analysant leur activité, on s'aperçoit qu'elles ont un certain nombre de ressources pour aider le visiteur à occuper chacune de ces classes de situation.

\section{Séquence 2 versus ACTIVITÉ ANALYTIQUE}

Au cours de la séquence 2, les animatrices insistent sur l'importance de partir de l'œuvre, de sa singularité pour faire décrire finement l'œuvre après avoir recueilli le ressenti des visiteurs et avant d'introduire des notions éventuellement plus théoriques. "Nous, notre singularité c'est qu'on a des auvres, voilà, on a des auvres et on est avant tout dans le sensible. » Elles mobilisent plusieurs ressources telles que des déictiques, des questions (a) questions ouvertes et déictiques pour soutenir l'exploration visuelle fine des visiteurs.

L'animatrice (A.) dit aux visiteurs (V.) "Qu'est-ce que vous voyez?"

Réponses des V. se chevauchant plus ou moins :-Deux visages/La même personne, mais pas habillée pareil/ Deux chefs/ Un couple/Un européen et un indien

A. oriente les V. vers des éléments pertinents, marquants du tableau en les questionnant: "Est-ce qu'ils regardent au même endroit?" "Quelle est l'expression de leur visage »

Réponses des V. : "L'un s'ennuie, l'autre est détendu/Ces deux personnages sont père et fils »

A. résume et reformule : «Ce sont bien deux hommes, un est habillé en habits traditionnels et l'autre en costume occidental, les hommes portent des bijoux »

Extrait d'observation a1
A. pointe avec le doigt les pointures sur le tableau : Comment savez-vous que cet homme est indien?

V. : Ces vêtements sont colorés

À : En quoi sont-ils faits?

V. : En peau d'animal

V.: Y'a des dessins et des couleurs

A. : C'est une peau de quoi?

V. : D'ours

V. : Ours polaire, Grizzly

À : Quels sont les autres indices?

V. : Les tipis

Extrait d'observation a2

Les animatrices nous ont également précisé en entretien d'auto-confrontation qu'une manière de faire rentrer le public dans l'œuvre consiste à leur demander de mimer par exemple la baigneuse de Picasso, elles demandent aux enfants de mettre la main gauche sur le ventre, pour des visiteurs plus âgés, elles leur demandent de décrire précisément la position des membres. Enfin, comme le montre l'extrait d'observation a1, parfois l'animatrice synthétise, résume, reformule et propose des interprétations ou des mots.

Les animatrices précisent qu'un des obstacles de cette classe de situation serait d'utiliser l'œuvre comme une illustration, ce qui appauvrirait l'œuvre, la réduirait à une illustration :

«Si on voit Aurélie Nemours, je ne vais pas commencer en disant ben voilà l'abstraction ... non! on va commencer par voir l'œuvre, on commence par le ressenti du public, la description fine puis des éléments sur l'œuvre en général de l'artiste, sa démarche et après, enfin moi je le prends dans ce sens-là, mais je préfère partir de ce que l'on a devant les yeux et on prend du recul petit à petit parce que voilà, si tu pars en disant Aurélie Nemours est une artiste qui s'inscrit dans l'abstraction, heu, géométrique à la suite de Mondrian, ... 
l'œuvre qu'on a sous les yeux perd de sa singularité, les gens vont se dire, ben oui, c'est un exemple d'abstraction, point! et ils vont chercher à retrouver ce que tu as dit »

Extrait d'entretien d'auto-confrontation animatrice

Ainsi la classe de situation description fine correspond à une séquence bien identifiée par les animatrices. Elles aident le visiteur à explorer l'œuvre singulière de manière prolongée, à caractériser, nommer, décrire ce qu'ils voient, ils peuvent s'asseoir devant l'œuvre, se reculer, s'avancer, mettre en rapport des éléments au sein de l'œuvre, mimer les divers éléments du tableau.

\section{Séquence 3 versus DISTANCIATION et ÉMERGENCE DE QUESTION}

Au sein de la troisième séquence, les animatrices ne distinguent pas explicitement les classes de situation distanciation et émergence de questions, en effet, au sein de leur activité ces classes de situations ont été intriquées. Nous les distinguons donc artificiellement pour clarifier notre propos :

Concernant la distanciation, l'animatrice introduit de nombreuses ressources telles que des ouvrages littéraires, historiques pour reconstruire le contexte de création (a), pour relier l'œuvre à des connaissances issues de diverses disciplines, domaines, pour introduire des concepts de l'histoire de l'art(b). L'animatrice outille le visiteur et explicite des techniques de l'art.

«Avoir un lien avec la littérature la musique de l'époque, d'autres artistes, et puis l'histoire aussi de l'époque parce que parfois on nous demande, mais c'était quel roi au $16^{\mathrm{e}}$ siècle donc voilà, donc on essaie chaque fois de faire le lien avec ce qui se passe dans le siècle »

«Faire comprendre au public comment est construite une image... ce sont des choix de composition, des décisions artistiques donc on essaie d'attirer l'attention sur les choix de composition, les intentions artistiques, les procédés. »

(a) Extraits d'Entretien d'auto-confrontation d'animatrices

A. : « Je voudrais que vous regardiez ce paysage ». A. pointe du doigt, A. « on voit quoi ?»

Plusieurs V. : «Le ciel/ la montagne/ on a l'impression qu'il y a du brouillard »

A. : « au loin, il y a plus de blanc, on n'a pas les contours très nets, en peinture on parle de perspective atmosphérique.»

(b) Extrait d'observation

Concernant la classe de situation d'émergence de question, les animatrices sont attentives tout au long de la troisième séquence à ne pas assener une interprétation définitive, elles essaient de pointer les divers éléments pertinents et laisse le visiteur en faire leur interprétation en fonction de ce qu'ils veulent y apporter (c).

«Quand on donne plein de réponses d'emblée, je ne sais pas ce qui reste, tandis que si on soulève des choses, sans apporter forcément de réponses, ça peut être plus intéressant et captivant à la fin, surtout les ados si on ne le met pas en position de s'interroger, ils ne vont pas accrocher »

«Le public accroche quand il sent la problématique sans se le dire, quand il peut poser des questions plus que quand on lui donne des réponses »

« Passer un bon moment, impliquer le visiteur transmettre des connaissances, mais surtout des questionnements, qu'ils se sentent enrichis et en même temps qu'ils aient apporté quelque chose. »

(c) Extraits d'entretien d'auto-confrontation

Par ailleurs, précisons que même si elles sont rares, des itérations sont possibles entre les 
trois séquences d'activité de l'animation (faire observer/faire décrire/faire analyser), c'est notamment ce que les animatrices mettent en œuvre pour casser certains préjugés de visiteurs et s'ajuster au public. L'animatrice synthétise et reformule en nuances des ressentis des visiteurs. "On essaie de partir de ce qu'ils connaissent pour les amener à ce qu'ils ne connaissent pas.»

\subsection{4.- Discussion}

Ainsi l'observation globale identifiée dans l'activité des animatrices consiste à amener les visiteurs à occuper la famille d'activité sensitive sans distinction spécifique des trois classes de situation (immersion, imprégnation, imagination). En effet, les animatrices n'incitent pas les visiteurs à occuper systématiquement chacune de ces trois classes de situation devant chaque œuvre. Ce qui prédomine pour les animatrices c'est d'octroyer un espace à la logique de subjectivation du visiteur.

En revanche, elles sont attentives à ce que les visiteurs occupent les trois classes de situation de la famille d'activité analytique devant chaque œuvre. Cette famille d'activité est soustendue par une logique d'objectivation, elles outillent les visiteurs à travers leurs questions pour la description fine, et de concepts et références pour la distanciation. Enfin, tout au long de la présentation de l'œuvre, elles privilégient l'émergence de questionnements de la part des visiteurs.

Notons que les animatrices n'adoptent pas la même attitude selon les classes de situation. Ainsi elles sont particulièrement à l'écoute des visiteurs, en les invitant à s'exprimer à propos de la famille d'activité sensitive et de la classe de situation d'émergence de questions, tandis qu'elles pointent des éléments, indiquent et alimentent les visiteurs grâce à leurs connaissances de manière beaucoup plus marquée en situation de description fine, et de distanciation. Les deux dimensions sensitives et analytiques de rencontre avec l'œuvre d'art s'organisent donc autour de critères différents : d'une part le point de vue subjectif du visiteur et d'autre part le point de vue «objectif» stabilisé des experts en histoire de l'art. Ces familles d'activité sont organisées de manière séquentielle lors des visites thématiques. Cependant, dans le cas de visites libres, nous pouvons émettre l'hypothèse que seules certaines facettes sont présentes, et qu'elles ne se déroulent pas toujours dans le même ordre. Ainsi si les animatrices aident les visiteurs à occuper les différentes classes de situation, on peut émettre l'hypothèse qu'un sujet capable tel que défini par Rabardel (2005) serait un visiteur susceptible, si l'œuvre lui plait, d'occuper et de se déployer au sein de ces différentes classes de situation.

\section{2.- Activité de visite muséale et médiation culturelle technique et individuelle}

\subsection{1.- Objectifs}

Nous avons mené une série d'expérimentations ergonomiques au sein du musée des beauxarts de Rennes où les visiteurs équipés d'un guide en réalité augmenté étaient amenés à effectuer une visite individuelle de 45 minutes environ. Nous cherchions à comprendre la manière dont ce guide augmenté transforme l'activité des visiteurs et à identifier les schèmes d'utilisation associés au guide augmenté. Notre objectif ici est d'utiliser le modèle de l'activité du visiteur capable pour éclairer la manière dont la réalité augmentée empêche et/ou privilégie certaines facettes de l'activité.

\subsection{2.- Méthodologie}

Les visiteurs étaient recrutés grâce à une petite annonce sur le site du Musée des Beaux-Arts de Rennes ainsi qu'une annonce sur un site de testeurs technophiles. Les visiteurs étaient équipés de caméra subjective fixée sur la tête pour enregistrer la visite dans sa progression dynamique. À l'issue de la visite, nous menions des entretiens individuels composés d'une partie relative à leurs pratiques muséales, une partie d'entretiens d'auto-confrontation et une 
partie consacrée à l'évaluation des fonctions du guide. Nous nous centrons ici sur la seconde expérimentation. 16 participants de 15 à 30 ans ont utilisé ce guide en situation de visite. Ils correspondent à la cible des visiteurs du musée envisagée pour ce guide.

Les visiteurs déambulaient librement et individuellement au sein du musée en utilisant le guide augmenté afin de réaliser une visite thématique sur le thème de l'inspiration. Le guide augmenté fournissait des indices pour trouver les 10 œuvres du parcours thématiques, ainsi que des informations permettant de souligner les différentes facettes de l'inspiration artistique grâce à la mise en évidence de détails, de points communs, et d'éléments d'histoire de l'art. Le guide augmenté est déployé sur une tablette. Pour accéder à l'information numérique, les visiteurs devaient se positionner face à la bonne œuvre et cadrer l'œuvre avec la tablette qui filme l'environnement réel (Figure 3). Lorsque le visiteur cible une œuvre pertinente, des contenus numériques s'affichent sur l'écran de la tablette comme le montre la figure 3 ci-dessous.

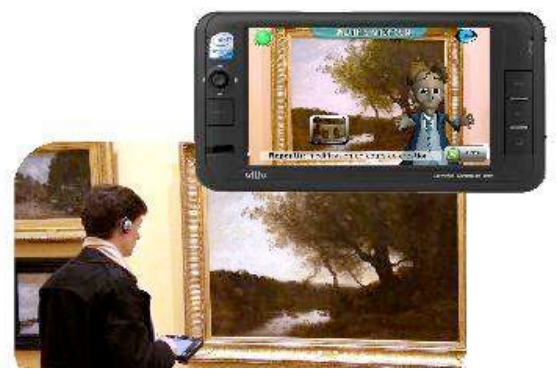

Figure 3 : Un visiteur utilisant le guide augmenté au musée de Beaux-Arts de Rennes

Figure 3: A visitor with the augmented reality museum guide in front of a painting

\subsection{3.- Guide augmenté et activité des visiteurs}

Nous présentons dans un premier temps l'organisation invariante de l'activité des visiteurs, autrement dit les schèmes d'utilisation des visiteurs. Nous détaillons dans un second temps, des séquences d'activité pour lesquelles la réalité augmentée est une ressource adéquate.

\section{Les schèmes d'utilisation}

L'accrochage correspond aux moments où le visiteur cadre une œuvre à l'aide de son guide augmenté. La déambulation des visiteurs est fragmentée dès lors que le nombre d'accrochages se multiplie.

\begin{tabular}{|l|l|l|l|}
\hline & $\begin{array}{l}\text { Nombre moyen } \\
\text { d'accrochages }\end{array}$ & $\begin{array}{l}\text { Nombre minimum } \\
\text { d'accrochages }\end{array}$ & $\begin{array}{l}\text { Nombre maximum } \\
\text { d'accrochages }\end{array}$ \\
\hline $\begin{array}{l}\text { Au cours d'une visite } \\
\text { d'environ 50 minutes }\end{array}$ & 38,08 & 14 & 80 \\
\hline
\end{tabular}

Tableau 2 : Nombre d'accrochages moyens, minimum et maximum au cours de la visite

Table 2: Number of framings mediated by the device during a visit (mean, minimum, maximum)

Ce tableau montre l'importance de la fragmentation de l'exploration muséale. Ainsi le visiteur qui a effectué 80 accrochages, est constamment derrière son dispositif pendant ses déambulations au sein du musée. Cependant la grande variabilité révèle des stratégies différentes de la part des visiteurs, c'est ce que nous allons voir grâce à l'identification des schèmes d'utilisation qui proviennent de la sphère des activités photographiques et vidéastes. 
Nous avons identifié trois schèmes d'utilisation de ce guide augmenté :

— Le schème de cadrage vidéo : ce schème est prédominant pour 5 visiteurs. Lorsque le visiteur mobilise ce schème, il se déplace dans le musée en regardant à travers le guide augmenté en continu. Le rapport aux œuvres est toujours médiatisé par la technique. Le sujet est amputé de son regard singulier. Les conséquences sont un appauvrissement de l'exploration visuelle à l'œil nu, un effet tunnel important, et in fine un primat est accordé au dispositif technique.

— Le schème de cadrage photographique en rafale : ce schème est mis en œuvre chez 7 visiteurs. Lorsque le visiteur mobilise ce schème, il regarde d'abord les œuvres à l'œil nu pour les localiser dans l'espace puis effectue des cadrages de ces œuvres avec le guide augmenté. L'exploration visuelle permet de repérer la présence des œuvres, mais l'œuvre est ensuite explorée de manière médiatisée. Le sujet se retrouve donc amputé de son libre arbitre. Les conséquences sont les suivantes : le regard est sanctionné, fragmenté par le dispositif technique, ainsi que l'exprime un visiteur: "quand je cherche le point rouge, j'ai réfléchi à l'inverse, je me demande vers quel tableau on va m'emmener. Je ne me suis pas demandé vers lequel moi j'irais, mais vers lequel on essaie de m'envoyer. J'essaie plusieurs tableaux.»

— Le schème du cadrage photographique modéré : ce schème est majoritairement mis en œuvre par 4 visiteurs. Le visiteur regarde les œuvres à l'œil nu (large exploration visuelle) et effectue seulement quelques prises avec le guide augmenté. Le dispositif fournit des indices au visiteur qui explore ensuite son environnement en fonction de ces indices. Le sujet est capable de découvrir des détails pertinents en autonomie. Le dispositif enrichit et oriente l'exploration visuelle. Ce schème semble approprié à l'activité de visite muséale dont l'objet de l'activité est de contempler les œuvres d'art.

Nous voyons ici qu'en fonction du schème d'utilisation, les visiteurs se rapprochent ou s'éloignent de la réalité. Au regard des principes pédagogiques muséaux, le schème du cadrage photographique modéré est le plus adapté au contexte muséal. Tandis que les deux autres schèmes opèrent un morcellement de l'activité et privent le visiteur d'une exploration pilotée par ses propres critères ou désirs. Ce résultat a permis d'orienter le cycle de conception afin de bloquer les deux schèmes inappropriés.

\section{Réalité augmentée et famille d'activité}

$\mathrm{Au}$ vu des caractéristiques du guide augmenté et des schèmes d'utilisation, nous identifions les classes de situation où le guide augmenté constitue un obstacle et/ou une ressource pour l'activité sensitive et analytique. Nous illustrerons nos propos en décrivant plus précisément quelques séquences d'activité.

\section{Guide augmenté et activité sensitive}

L'activité sensitive nécessite un temps long d'observation pour s'immerger, s'imprégner ou imaginer or les schèmes photographiques fragmentent cette observation globale. À ce propos, Sontag (2000) qui a étudié la prise photographique explique que «le cadrage photographique peut provoquer un éloignement par rapport à la nature, plutôt qu'une union avec elle». Ainsi, la réalité augmentée semble faire entrave à l'activité d'immersion, d'imprégnation et d'imagination, en tous cas la fragmente. Ce morcellement n'est pas compatible avec un rapport global et continu avec l'œuvre où le visiteur se laisse envelopper, pénétrer par ses impressions, sensations, émotions et ressentis.

Cependant, malgré ce morcellement, certaines fonctions du guide augmenté semblent privilégier l'activité sensible.

Les fonctions sonores telles que la musique et le son constituent une ressource pour aider certains visiteurs à s'immerger et à imaginer l'œuvre plus vivante. Ainsi, un des visiteurs nous fait part de l'intérêt de l'extrait musical provenant du guide augmenté et associé à une œuvre "c'est intéressant ça fait un paysage sonore, ça peut compléter l'ambiance, là je m'imprègne». Voici ce qu'un autre visiteur nous dit à propos du cri du tigre associé à $l a$ 
Chasse au tigre de Rubens : "le cri du tigre rend le tableau vivant». Ces remarques n'ont pas été majoritaires, mais tout de même pointées par un tiers des visiteurs.

De la même manière, des éléments graphiques du guide augmenté peuvent aider le visiteur à s'immerger dans l'œuvre. La composition ajoutée sur l'œuvre de Rubens grâce au guide augmenté (cf. Figure 4) peut aider le visiteur à organiser son expérience sensitive. Ainsi, un visiteur nous a dit, que se trouvant en face du tableau de Rubens, il ressentait une impression de courbe, mais qu'il ne pouvait pas l'exprimer précisément avant de voir la composition en spirale sur le guide augmenté.

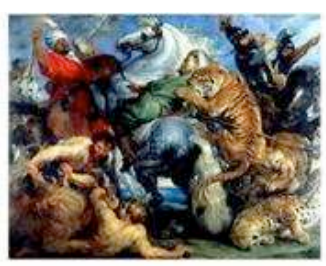

(1)

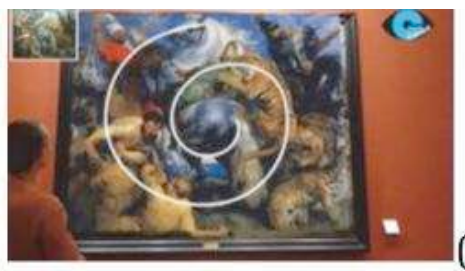

(2)

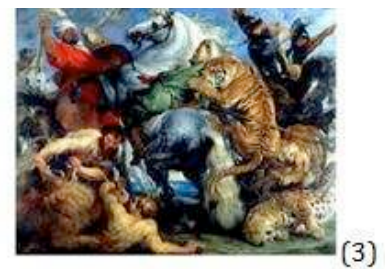

Figure 4 : Séquence d'activité : immersion (1) - distanciation(2) - l'immersion (3)

Figure 4: Sequence of activity: immersion (1)-objectification (2)-immersion (3)

Cette situation nous permet donc de décrire la séquence d'activité du visiteur: (1) il contemple l'œuvre d'art en écoutant ses sensations (il ne sait pas mettre de mots), (2) il regarde le guide augmenté qui présente la composition du tableau en spirale, (3) il regarde à nouveau l'œuvre, à ce moment-là il est capable de mettre le mot de courbe sur sa sensation face à l'œuvre. Il s'agit donc d'une séquence d'activité qui enchâsse la distanciation médiatisée entre deux phases d'immersion non médiatisées.

Ainsi l'activité sensitive n'est pas spécialement privilégiée par ce guide augmenté, même si nous avons identifié des situations précises où le son, la musique et les représentations graphiques de la composition pourraient être des ressources pour aider le visiteur à mieux s'immerger ici et maintenant dans l'œuvre et à mieux imaginer la scène en mouvement. En revanche, l'imprégnation n'est pas du tout outillée par le guide augmenté.

\section{Guide augmenté et activité analytique}

Nos analyses mettent en évidence une prédominance du guide augmenté pour outiller la description fine de l'œuvre ainsi que pour aider le visiteur à analyser l'œuvre de manière ludique grâce aux manipulations rendues possibles (distanciation).

Toutefois, la réalité augmentée privilégie une approche analytique de l'œuvre, si et seulement si l'incorporation de la réalité est effectuée. En effet, les éléments d'analyse enrichissent la visite muséale uniquement s'ils concourent à inciter le visiteur à observer l'œuvre réelle. La situation de mise en évidence du repentir de l'œuvre de Corot grâce à la loupe du guide augmenté est un exemple frappant (cf. Figure 5).

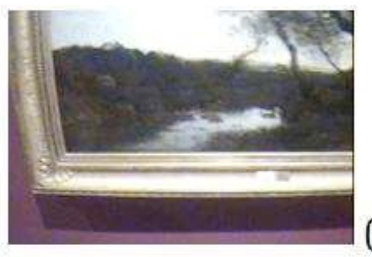

(1)

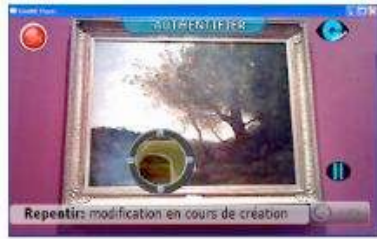

(2)

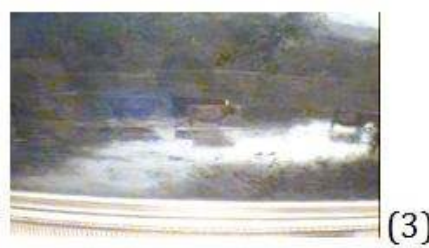

Figure 5 : Séquence d'activité : description fine (1)-distanciation(2)-description fine (3)

Figure 5: Sequence of activity: detailed description (1)-objectification (2)detailed description (3) 
Dans un premier temps, le visiteur regarde l'œuvre à l'œil nu (1), dans un second temps le visiteur utilise l'outil loupe sur le guide augmenté pour chercher le repentir (2). Une fois repéré sur le guide augmenté, lorsque le visiteur regarde la toile à l'œil nu, il découvre les traces du repentir (3).

Cette tâche a été très appréciée par treize visiteurs sur seize, tous ont souligné l'importance de l'aller-retour entre le guide augmenté et l'œuvre réelle :

« j'ai utilisé la loupe pour regarder dans le tableau, au milieu du tableau je me suis dit que c'était l'endroit le plus propice pour un élément caché puis en descendant plus bas, je suis tombé sur la rivière et j'ai trouvé le pont. Ensuite j'ai regardé dans le vrai tableau pour voir le pont qui ne m'avait pas du tout sauté aux yeux la première fois et là je le voyais très bien ».

« je parcours l'œuvre et je vérifie que ça correspond et puis heu... je le cherche, j'essaie de voir effectivement ce qui reste sur l'œuvre réelle de ce que je vois avec la loupe, je m'approche et c'est assez bluffant de découvrir un détail qui n'était pas visible au début »

« là j'ai vraiment aimé...j'ai appris quelque chose et de le voir en s'approchant du tableau $» . .$.

«C'était bien de faire le lien entre le virtuel et le réel »

Cette situation de recherche du repentir est une séquence d'activité typique qui alterne la classe de situation: description fine (exploration de l'œuvre singulière)/distanciation (présentation du concept de repentir et des indices sur le guide)/description fine (exploration visuelle de l'œuvre singulière). Il existe un aller-retour entre la posture du visiteur en description fine non médiatisée par le guide augmenté et distanciation médiatisée par le guide augmenté. Cette séquence est typique des séquences qui ont été les plus appréciées par les visiteurs. Leurs caractéristiques communes étaient une alternance de la description fine (exploration œuvre(s) à l'œil nu) et de la distanciation (présentation indices/jeux sur guide).

En contrepoint, les séquences d'activité qui n'incluaient pas cet aller-retour entre description fine et distanciation en proposant des vidéos de 2 minutes relatives à l'artiste d'une œuvre par exemple ont été perçues comme trop longues. De la même manière, une séquence d'activité qui révélait la présence d'un repentir sur l'œuvre de Chardin a peu intéressé les visiteurs dans la mesure où ils ne pouvaient ensuite vérifier directement sur l'œuvre, à l'œil nu, le nouvel élément d'information.

Le guide augmenté est donc une ressource pour le visiteur lorsqu'il y a une alternance de séquences de description fine non médiatisée et distanciation médiatisée. Précisons, qu'il outille peu la classe de situation émergence de questions.

\subsection{4.- Discussion}

Un visiteur résume les caractéristiques du guide augmenté de la manière suivante : «L'outil fait davantage tendre vers une pensée analytique et c'est à chacun selon ses propres ressources d'en venir à une lecture plus globale». Il s'agit donc pour les concepteurs d'inviter le sujet à alterner les séquences d'exploration de l'œuvre à l'œil nu et d'exploration médiatisée. En effet, la distanciation est une classe de situation qui consiste à relier l'œuvre avec d'autres domaines et sa combinaison avec l'immersion et la description fine qui se situent dans le ici et maintenant est fructueuse. Dans cette perspective, la réalité augmentée pourrait être envisagée dans les termes d'une « technologie trouée » à utiliser dans les phases de distanciation comme une dentelle qui tisse le réel du visiteur dans les situations ici et maintenant sans lui substituer une tout autre réalité. Elle est donc pertinente pour les allersretours entre description fine et distanciation et pourrait l'être entre immersion et distanciation. Ainsi, il s'agit d'un enrichissement entre classes de situation. En revanche, le guide augmenté outille peu l'imprégnation, l'imagination et l'émergence de questions.

Dans une perspective muséale, la réalité augmentée doit donc disparaître dans les phases d'activité sensitive et de description fine pour n'apparaître que ponctuellement pour outiller 
l'activité de distanciation. Ceci nécessite de penser en amont une situation de visite laissant des temps à l'activité sensitive. Dans le cadre de ce projet, la fonctionnalité de réalité augmentée a donc été bloquée dans les phases de déambulation au sein du musée, et circonscrite aux phases d'exploration d'une œuvre une fois l'énigme posée pour inviter le visiteur à renouveler son regard sur l'œuvre .

Le tableau 3 ci-dessous résume ces conclusions.

\begin{tabular}{|l|l|l|}
\hline & Activité sensitive & Activité analytique \\
\hline Ici et maintenant & Immersion non médiatisée & Description fine non médiatisée \\
\hline relier & & Distanciation médiatisée \\
\hline
\end{tabular}

Tableau 3 : Adéquation entre le guide augmenté et les classes de situation du modèle MARO

Table 3: Coherency between the augmented reality guide and the categories of situation in the MARO model

\section{5.- Quels sont les apports du modèle de l'activité du sujet capable?}

Nous organisons la discussion en trois axes, nous aborderons tout d'abord le modèle de l'activité et ses prolongements possibles, nous nous questionnerons ensuite sur le rôle de ce modèle pour le domaine muséal, enfin nous terminerons en nous questionnant sur le statut de ce modèle au sein de l'ergonomie.

\section{1.- Les prolongements de MARO}

Le modèle MARO en mettant en évidence les deux familles d'activité analytique et sensitive, révèle le faible nombre d'études relatives aux pratiques muséales qui éclairent plus précisément l'activité sensitive.

À ce titre, l'activité sensitive mériterait d'être creusée de manière empirique. Cependant, pour approcher les activités sensitives, il semble important de distinguer le comportement observable et les mouvements de l'expérience. Nous rejoignons Bachelard (1943) qui souligne que l'étude objective et visuelle du changement - étude toute cinématique - ne permet pas d'approcher le mouvement de l'expérience, notamment les échos que provoquent l'œuvre. Ainsi le visiteur lorsqu'il se trouve dans cette posture de subjectivation, s'utilise comme caisse de résonnance et écoute, dirige, organise les échos que l'œuvre provoque en lui. Cette famille d'activité sensitive pourrait donc être approfondie notamment à l'aide de la technique de l'entretien d'explicitation (Vermersch, 1994) afin de documenter plus précisément les trois classes de situation: immersion, imprégnation et imagination. C'est d'ailleurs le travail initié par Petimengin (2007) qui décrit avec précision les fragments de vécu préréflexif que ressentent les personnes en la présence d'œuvre d'art: «l'impression globale et complexe», «vibrations qui viennent toucher des zones difficiles à situer impressions fugaces d'intensité, de contraste et de résonnance, transmodales », d'une expérience intérieure, non déterminée sensoriellement. Elle pointe la perméabilité entre le monde extérieur et intérieur ainsi que la présence de sensations et de vibrations qui émergent dans un espace qui n'est ni subjectif, ni objectif. Ce travail pourra donc être prolongé en documentant de manière plus systématique les classes de situation d'immersion, mais également d'imprégnation et d'imagination.

Le modèle MARO constitue également une base intéressante pour questionner l'articulation des activités productives et constructives du visiteur capable (Rabardel, 2005) en contexte muséal. En effet, lorsque l'art fonctionne, l'œuvre éveille, élargit la vision des visiteurs en 
apportant des connexions et des contrastes nouveaux (Goodman, 1996). Lorsqu'il s'agit d'un choc émotionnel, d'une rencontre véritable avec une œuvre, nous pensons que le visiteur élabore de nouvelles ressources et réorganise son rapport au monde, il s'agit alors de la dimension constructive de l'activité (Rabardel, 2005). Il serait intéressant de comprendre comment ces ressources s'élaborent au sein de chacune des classes de situation, et de décrire la manière dont le sujet devient capable de se déployer dans chacune ces classes de situation.

Pour finir, il sera également important de continuer de mettre à l'épreuve ce modèle au sein d'une collection de situations de médiation culturelle diversifiées afin d'estimer la résistance des classes de situation, ainsi que la dimension heuristique de ces catégories pour décrire l'activité réelle telle qu'elle se déploie dans ses dimensions sociales. Nous avons d'ores et déjà initié ce travail au sein d'un projet collaboratif avec plusieurs musées d'art parisiens.

\section{2.- Le statut de ce modèle pour les professionnels du musée et les implications pour la conception}

Dans un premier temps, nous montrons de quelle manière le modèle MARO a nourri nos échanges avec les professionnels du Musée des Beaux-Arts de Rennes, dans un second temps, nous évoquons son utilité pour le domaine muséal plus largement.

À partir des deux études empiriques au Musée des Beaux-Arts, le modèle révèle la complémentarité possible de ces deux types de médiation culturelle : la visite thématique guidée permet au visiteur face à l'œuvre d'occuper la famille d'activité sensitive dans un premier temps puis de se déployer dans toutes les classes de situation analytique de manière systématique dans un second temps. Le guide augmenté privilégie les séquences d'activité composées de description fine et de distanciation. Il pourrait également être une ressource pour outiller les séquences d'activité constituées d'immersion et de distanciation de manière plus systématique au cours de visites à créer en fonction de ce que les animatrices ont envie de faire toucher du doigt au public. Le modèle permet d'alimenter la réflexion des animatrices concernant la complémentarité de leurs dispositifs de médiation culturelle ainsi que de pointer des types de visites augmentées à concevoir dans le futur. À l'issue de la recherche, la dimension opératoire de ce modèle de l'activité a été soulignée par les animatrices du musée des Beaux-Arts de Rennes. En effet, ce modèle fait sens dans le champ de leurs pratiques de la médiation culturelle. D'une part, il constitue une ressource pour former les stagiaires et nouvelles recrues à leur démarche. D'autre part, malgré la multitude d'études traitant des visiteurs de musée, elles ont des difficultés à les articuler les unes aux autres ainsi qu'à leurs pratiques en situation. La description des séquences d'activité des visiteurs semble être opératoire pour leur pratique.

Lors de nos divers présentations et échanges avec des responsables de services des publics, il apparaît que ce modèle (cf. Figure 6) pourrait être également utilisé comme une cartographie permettant de positionner les différents dispositifs de médiation d'un musée ainsi que de rendre visible le travail effectué par les médiateurs auprès de la hiérarchie des institutions culturelles. En effet, ces services n'échappent pas à la nécessité de justifier leur productivité en termes quantitatifs (Edeilman, \& Roustan, 2007). Ce type de modèle est donc une ressource pour déplacer le débat de la productivité quantitative vers les caractéristiques des dispositifs de médiation mis en place. Plus précisément, ce modèle qui présente les diverses facettes de la rencontre avec l'œuvre d'art peut participer à éclairer au sein des institutions muséales la spécificité des médiateurs qui intègrent le vécu sensitif des visiteurs. Il constitue également une ressource pour les services des publics pour concevoir, évaluer et rendre visible les dispositifs de médiation culturelle et leurs complémentarités. La mise en visibilité de cette activité sensitive, ainsi que l'élaboration de ressources au service de cette dernière constitue un enjeu identitaire important pour les médiateurs qui travaillent aussi bien la capacité analytique que sensitive que les visiteurs déploient dans leur relation à l'œuvre d'art. 


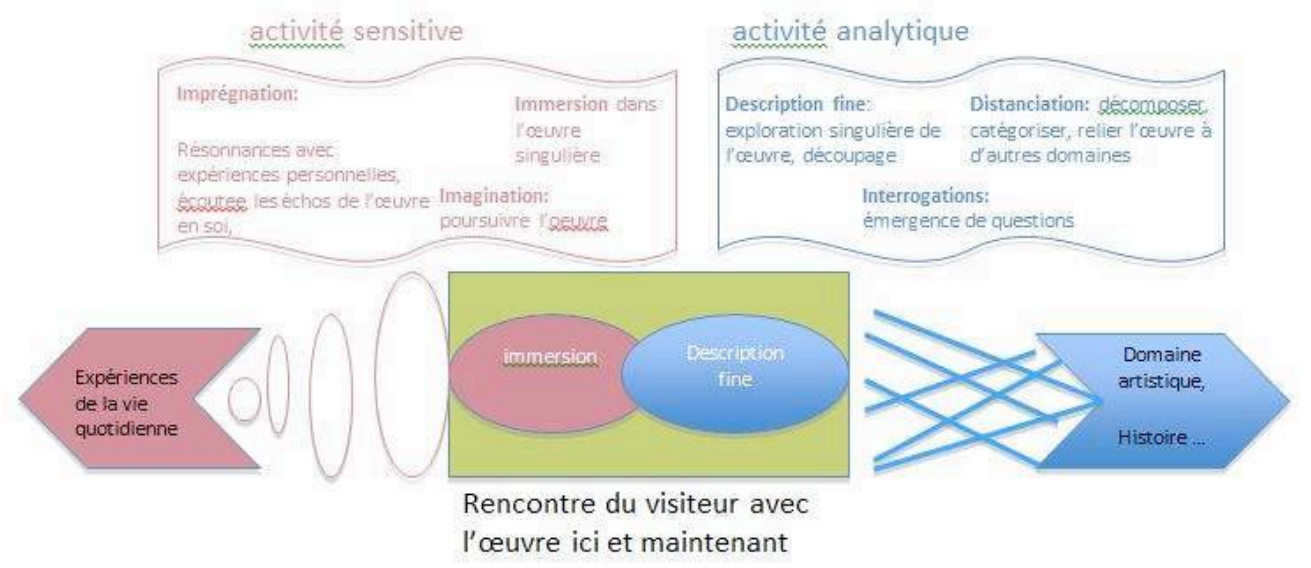

Figure 6 : Le Modèle d'Activité de Rencontre avec une Oeuvre en contexte muséal (MARO)

Figure 6: The Model MARO (activity of encounter with a work of Art in a museum)

\section{3.- Le statut de ce modèle dans une perspective de généralisation scientifique}

Nous proposons un modèle de l'activité du visiteur capable. Cette proposition nous semble intéressante sur le plan scientifique, et pragmatique.

En effet, concernant la recherche, le modèle de l'activité du sujet capable est une voie intéressante pour monter en généralisation. Cependant, le modèle d'activité du sujet capable n'a pas pour vocation de se réifier. Il doit être réinterrogé en fonction des évolutions caractéristiques des visiteurs, des milieux muséaux et des objectifs de l'animation. C'est ce que nous entreprenons au sein d'une diversité de projets culturels dans lesquels nous sommes insérée dans le cadre de la conception de guide (musée d'art, musée des sciences, musée d'art contemporain, etc.) ou de travail collaboratif avec les services des publics de plusieurs musées. Ainsi, nous pourrons comparer une myriade d'études de cas, les unes aux autres grâce à ce modèle d'activité afin de documenter et généraliser les invariants de l'activité, afin de préciser les ressorts de chacune des classes de situation. Nous nous inscrivons donc dans la perspective de Daniellou (1996) qui souligne les allers-retours nécessaires entre les modèles de l'activité et le réel.

En proposant un modèle de l'activité du sujet capable, nous proposons un écart heuristique entre activité réelle et activité du sujet capable. Cette démarche n'est pas nouvelle puisque l'ergonomie vise depuis de nombreuses années à rendre compte de l'écart entre le prescrit et le réel. Il a d'ailleurs été souvent montré que le réel dépasse le prescrit. Pouvoir confronter l'activité réelle à l'activité du sujet capable est une démarche complémentaire qui permet de révéler la manière dont les situations actuelles pourraient être reconçues, réorganisées pour que l'activité se déploie. Cet écart entre l'activité réelle et l'activité du sujet capable nous semble donc une voie complémentaire pour développer une ergonomie de prospective.

Enfin, le modèle d'activité du sujet capable permet un changement de posture de l'ergonomie notamment dans le champ de la conception des nouvelles technologies. Au sein de ces projets, l'ergonome est souvent cantonné à un rôle d'évaluateur. Avec un modèle de l'activité du sujet capable, il acquiert une posture qui consiste à détecter au sein des situations les gisements à exploiter et à faire grandir pour augmenter le pouvoir d'agir dans une situation singulière. Plus précisément, ce modèle permet de proposer une vision alternative aux spécialistes de réalité augmentée qui ont tendance à penser l'utilisateur augmenté comme un utilisateur en mesure de traiter une profusion d'informations toujours 
plus nombreuses. Or cette étude révèle la nécessité pour le visiteur d'alterner une exploration augmentée et une exploration à l'œil nu. Dans ce contexte, la réalité augmentée semble plus pertinente quand elle réduit la complexité de la réalité pour orienter le visiteur dans une exploration renouvelée de son environnement. Dans cette perspective, la réalité augmentée peut fournir des images opératives (Ochanine, 1978) externalisées. Lorsque le visiteur utilise une loupe, il accède bien à une représentation lacunaire de la réalité, voire déformée, mais opérationnellement adaptée pour identifier un repentir. Cette manière de scruter les œuvres peut ensuite être internalisée par les visiteurs. Ainsi, la réalité augmentée permet de transformer la manière de considérer la réalité à un moment donné, mais peut également soutenir le développement de la perception du visiteur.

Dans une perspective d'éducation muséale, l'écart entre l'activité réelle et l'activité du visiteur capable révèle la zone potentielle de développement des visiteurs. La qualification fine de cette zone potentielle de développement peut donc orienter les médiateurs pour concevoir de nouvelles situations d'éducation muséale en combinant la multiplicité des ressources à leur disposition (ateliers, cartels, audioguides, visite guidée, muséographie, espace).

Il en découle un certain nombre de questionnements qui dépassent le champ muséal que nous formulons de la manière suivante: comment concevoir des situations, des outils qui permettent à l'homme de déployer aussi bien la dimension analytique et sensitive de ses activités ? Comment concevoir des technologies trouées à la manière des blancs de la narration $(E c o, 1985)$ afin que le sujet capable puisse se projeter ou s'imprégner des situations?

Remerciements :

Je remercie Françoise Decortis pour tous les échanges passés, présents et futurs sur ce thème ; Pierre Rabardel et Cécile Barbier pour leurs relectures toujours bienveillantes et leurs conseils ; Marie B pour son œil de lynx. Je remercie également les financeurs et partenaires du projet ANR GAMME et plus particulièrement les animatrices et conservatrice du Musée des Beaux-Arts de Rennes (Odile, Anne-Sophie, Carole et Laurence) avec qui ce fut un plaisir de travailler au fil de ces trois années.

\section{BIBLIOGRAPHIE}

Azuma, R.T. (1997 ). A survey of augmented reality. Presence: Teleoperators and virtualEnvironments, 6, 355-385.

Bachelard, G. (1943). L'air et les songes, essai sur l'imagination du mouvement. Paris: Corti.

Bachelard, G. (1971). La formation de l'esprit scientifique. Paris: Vrin.

Bach, C., Salembier P., \& Dubois, E. (2007) Co-conception centrée utilisateur de systèmes interactifs pour les musées : application à la découverte de la cladistique. In M. Zouinar, G. Vallery, \& M.-C. Le Port (Eds.), Ergonomie des produits et des services médiatisés : nouveaux territoires, nouveaux enjeux (pp 69-78). Paris ): PUF.

Bationo-Tillon, A., Marchal, I., \& Houlier, P. (2009). When augmented reality enriches museum visit: Lessons learned. In SHIRAI Akilhido (Eds.). Proceedings of Virtual Reality International Conférence (VRIC 2010), pp. 227-234.

Bationo-Tillon A., Marchal I, \& Houlier P. (2010). A day at the museum. Proceedings of the ninth IEE International Symposium on Mixed and Augmented Reality, ISMAR 2010, Seoul, pp. 69-70.

Bationo-Tillon, A., Marchal I, \& Houlier P. (2011). Mobile Augmented Reality in the Museum: Can a lace like technology take you closer to works of art? Proceedings of the tenth IEE International Symposium on Mixed and Augmented Reality, ISMAR 2011, Bâle, pp. 41-47.

Belcher, M. (1991). Exhibitions in museums. London and New York: Leicester University Press.

Caillet, E. (1994). L'ambiguité de la médiation culturelle : entre savoir et présence. Public et musées, 6, 53-73.

Cohen, S. (1996). Children and adults. In G. Durbin (Ed.), Developping museum exhibitions for lifelong learning (pp.73-76). London: Museums and galleries Commission. 
Damala, A., Cubaud, P., Bationo-Tillon, A., Houlier, P., \& Marchal, I. (2008). Bridging the Gap between the Digital and the Physical: Design and Evaluation of a Mobile Augmented Reality Guide for the Museum Visit. DIMEA'08, 3rd Int. Conf. on Digital Interactive Media in Entertainment and Arts, Athens, Greece, September 2008, pp. 120-127.

Daniellou, F. (1996). L'ergonomie en quête de ses principes. Débats épistémologiques. Toulouse: Octarès.

Daniellou, F., \& Beguin, P. (2004). Méthodologie de l'action ergonomique : approches du travail réel. In P. Falzon (Ed.), Ergonomie (pp 335-358). Paris: PUF.

Deshayes, S. (2002). Audioguides et musées. La lettre de l'OCIM, 79, 24-31.

Dewey, J. (2010). L'art comme expérience. Paris: Gallimard.

Donat, O. (1998). Les pratiques culturelles des français. Enquête 1997. Paris: La Documentation Française.

Dufresne-Tassé, C. (2006). L'éducation muséale des adultes, un domaine à structurer. Savoirs, 11(2), 39-64.

Eco, U. (1985). Lector in Fabula. Paris: Grasset.

Edeilman, J., \& Roustan, M. (2007). Les études de publics : recherche fondamentale, choix de politiques et enjeux opérationnels. In J. Edeilman, M. Roustan \& B. Goldstein (Eds.), La place des publics : de l'usage des études et recherches par les musées (pp. 15-37). Paris: La documentation française.

Ekary, M. (1986). Combinating Redundancy : writing texts for exhibitions. Exibitions in Sweden, $27(8), 1-7$.

Falk, J., \& Dierking, L.D. (1992). The museum experience. Washington: Whalesback books.

Falzon, P. (1998). Qu'est-ce que la recherche en ergonomie ? Présenté colloque «Recherche et ergonomie », Toulouse, France, février 1998. http ://www .ergonomieself.org/media/media30493.pdf

Fauche, A. (2008). La médiation scientifique et culturelle de musée. Document de travail : juillet 2008. http ://www.ldes.unige.ch/publi/i_artVulg.htm

Fauche, A. (1998). Le médiateur culturel au musée : Quelle spécificité, quelle formation ? Actes JIES XIX, 1998. http://www.ldes.unige.ch/publi/i_artVulg.htm

Folcher, V., \& Bationo-Tillon, A. (2012). De l'interaction home-machine à l'activité médiatisée : apports et limites de la réalité augmentée en situation de visite muséale. Revue des Interactions Humaines Médiatisées, 12(2), 3-24.

Fockler,P., Zeidler, T., Brombach, B., Bruns, E., \& Bimber O. (2005). Phoneguide : Museum guidance supported by on-device object recognition on mobile phones. Proceedings of the 4th International Conference on Mobile and ubiquitous multimedia, 2005, pp. 3-10.

Goodman, N. (1996). L'art en théorie et en action. Paris : L'éclat.

Hindmarsh, C., Heath, C., \& Vom Lehn, D. (2005). Creating assemblies in public environments: social interaction, interactive exhibits and CSCW. Computer Supported cooperative work, 14(1), $1-41$.

Hooper-Greenhill, E. (1994). Museums and their visitor. London: Routledge.

Hooper- Greenhill, E. (1999). The educational Role of the museum. London: Routledge.

Hooper Greenhill, E., Moussouri, T., Hawtorne, E., \& Riley, R. (2001) Making meaning in Art museums 1: visitors' interpretive strategies at Wolverhampton Art Gallery. RCMG. http://www.le.ac.uk/museumstudies/research/rcmgpublicationsandprojects.html

Hope T., Nakamura Y., Takahashi T., Nobayashi, A., Fukuoka, S., Hamasaki,M. et al. (2009). Familial Collaborations in a museum. Proceedings of CHI 2009, pp. 1963-1972.

Liestol, T. (2009) Augmented reality and Digital Genre Design- Situated simulations on the iPhone". IEEE ISMAR 2009 AMH Proceedings, 19-22 october, Orlando, Florida, USA, pp. 29-34.

Lockett, C. (1991). Ten years of exhibit evaluation at the royal Ontario museum (198-1990). A journal of visitor behavior, 2(1), 19-46.

Mcmanus, P.M. (1989). Oh, yes they do: how museum visitors read labels and interact with exhibit texts. Curator. 32(3), 174-189. 
Miyashita, P., Meier, T., Tachikawa, S., Orlic, T., Eble, V., Scholz, A., et al. (2008). An augmented reality museum guide. Proceedings of the seventh International Symposium on Mixed and Augmented Reality, pp. 103-106.

Merleau Ponty, M. (1945). Phénoménologie de la perception. Paris: Gallimard.

Ochanine, D. (1978) Le rôle des images opératives dans la régulation des activités de travail. Psychologie et éducation, 3, 63-79.

Ombredane, A., \& Faverge, J.-M. (1955). L'analyse du travail. Paris: PUF.

Passeron, J.-C. (1974). L'euvre picturale et les fonctions de l'apparence. Paris: Vrin.

Pedler, E. (2005). En quête de réception : le deuxième cercle. Réseaux, 68, 85-103.

Peyrin, A. (2010). Etre médiateur au musée, sociologie d'un métier en trompe l'œil. Paris: La Documentation Française.

Peyrin, A. (2012). Focus : les paradoxes de la médiation culturelle dans les musées. Informations sociales, $170(2), 62-65$.

Petitmengin, C. (2007). Towards the source of thougths. The gestural and transmodal dimension of lived experience. Journal of consciousness studies, 14(3) 54-82.

Rabardel P. (1995). Les Hommes et les technologies, approche cognitive des instruments contemporains. Paris: Armand Colin. http://ergoserv.psy.univ-paris8.fr/

Rabardel, P. (2005). Instrument subjectif et développement du pouvoir d'agir. In P. Rabardel \& P. Pastré (Eds.), Modèles du sujet pour la conception (pp. 11-30). Toulouse: Octarès.

Rabardel, P., \& Bourmaud, G. (2003). From computer to instruments system: a developmental perspective. Interacting with computer, 15(5), 665-691.

Rabardel, P., \& Samurçay, R. (2001). Artifact mediated Learning. In Y. Engeström, \& M. Hasu (Eds.), New Challenges to research on Learning. Lawrence Erlbaum.

Rebetez, P. (1970). Comment visiter un musée? Strasbourg : Conseil de la Coopération Culturelle du Conseil et de l'Europe.

Sontag, S. (2000). Sur la photographie. Paris: « choix-essais » Christian Bourgois.

Theureau, J., \& Pinsky, L. (1984). Paradoxes de l'ergonomie de conception et logiciel informatique. Revue des conditions de travail, 9.

Tisseron, S. (2005). La réalité de l'expérience de fiction. L'Homme, 175-176(3), 131-145.

Vermersch, P. (1994). L'entretien d'explicitation. Issy Les moulineaux: ESF.

Véron, E., \& Levasseur, M. (1991). Ethnographie de l'exposition. Paris: Éditions de la Bibliothèque publique d'information.

Wakkary, R., Hatala, M., Muise, K., Tanenbaum, K., Corness, G., Mohabbati B., \& Budd, J. (2009). Kurio: a museum guide for families. Proceedings of the Third International Conference on Tangible and Embedded Interaction (TEI'09), pp. 215-22.

Woodruff, A., Szymanski, M.-H., Grinter, R.-E., \& Aoki, P. (2002). Practical Strategies for Integrating a Conversation Analystin an Iterative Design Process. IEE. 4th Conference. on Designing Interactive Systems, London, June 2002, pp. 19-28.

\section{RESUME}

Cet article propose d'aborder le domaine muséal à travers des concepts et méthodes issus de l'ergonomie. Après une revue de l'art succincte des questions qui traversent ce champ et celui de la médiation culturelle, nous proposons un modèle de l'activité de rencontre avec une œuvre d'art (MARO) d'un visiteur de musée capable. Plus précisément, nous examinons l'activité de rencontre avec une œuvre d'art, en éclairant les différentes classes de situation qui peuvent se déployer en contexte. Ce papier présente les résultats empiriques de deux études complémentaires : des activités muséales dans une situation de visite collective et guidée ainsi que dans un contexte de visite individuelle instrumentée. Nous décrivons et confrontons les invariants de l'activité des 
visiteurs au modèle MARO. Enfin nous discutons des apports de ce modèle de l'activité muséale du visiteur capable pour mieux étudier et outiller les activités de visite de musée, ainsi que les professionnels de musée.

\section{MOTS CLES}

sujet capable, médiation, œuvres d'art muséales

\section{REFERENCEMENT}

Bationo-Tillon, A. (2013). Ergonomie et domaine muséal. Activités, 10(2), 82-108, http://www .activites.org/v10n2/v10n2.pdf

Article soumis le 24 août 2012, accepté pour pulciation le 13 mai 2013. 